\title{
The EBLM project ${ }^{\star, \star \star, \star \star \star}$
}

\section{Physical and orbital parameters, including spin-orbit angles, of two low-mass eclipsing binaries on opposite sides of the brown dwarf limit}

\author{
A. H. M. J. Triaud ${ }^{1}$, L. Hebb ${ }^{2}$, D. R. Anderson ${ }^{3}$, P. Cargile ${ }^{2}$, A. Collier Cameron ${ }^{4}$, A. P. Doyle ${ }^{3}$, F. Faedi ${ }^{5}$, M. Gillon ${ }^{6}$, \\ Y. Gomez Maqueo Chew ${ }^{5}$, C. Hellier ${ }^{3}$, E. Jehin ${ }^{6}$, P. Maxted ${ }^{3}$, D. Naef ${ }^{1}$, F. Pepe ${ }^{1}$, D. Pollacco ${ }^{5}$, D. Queloz ${ }^{1}$, \\ D. Ségransan ${ }^{1}$, B. Smalley ${ }^{3}$, K. Stassun ${ }^{2}$, S. Udry ${ }^{1}$, and R. G. West ${ }^{7}$
}

\author{
1 Observatoire Astronomique de l'Université de Genève, Chemin des Maillettes 51, 1290 Sauverny, Switzerland \\ e-mail: Amaury.Triaud@unige.ch \\ 2 Department of Physics and Astronomy, Vanderbilt University, Nashville, TN37235, USA \\ 3 Astrophysics Group, Keele University, Staffordshire, ST55BG, UK \\ 4 SUPA, School of Physics \& Astronomy, University of St Andrews, North Haugh, KY16 9SS, St Andrews, Fife, Scotland, UK \\ 5 Astrophysics Research Centre, School of Mathematics \& Physics, Queens University, University Road, Belfast, BT71NN, UK \\ ${ }^{6}$ Institut d'Astrophysique et de Géophysique, Université de Liège, Allée du 6 Août 17, Bat. B5C, Liège 1, Belgium \\ 7 Department of Physics and Astronomy, University of Leicester, Leicester, LE17RH, UK
}

Received 22 May 2012 / Accepted 27 September 2012

\begin{abstract}
This paper introduces a series of papers aiming to study the dozens of low-mass eclipsing binaries (EBLM), with F, G, K primaries, that have been discovered in the course of the WASP survey. Our objects are mostly single-line binaries whose eclipses have been detected by WASP and were initially followed up as potential planetary transit candidates. These have bright primaries, which facilitates spectroscopic observations during transit and allows the study of the spin-orbit distribution of $\mathrm{F}, \mathrm{G}, \mathrm{K}+\mathrm{M}$ eclipsing binaries through the Rossiter-McLaughlin effect.

Here we report on the spin-orbit angle of WASP-30b, a transiting brown dwarf, and improve its orbital parameters. We also present the mass, radius, spin-orbit angle and orbital parameters of a new eclipsing binary, J1219-39b (1SWAPJ121921.03-395125.6, TYC 7760484-1), which, with a mass of $95 \pm 2 M_{\text {jup }}$, is close to the limit between brown dwarfs and stars. We find that both objects have projected spin-orbit angles aligned with their primaries' rotation. Neither primaries are synchronous. J1219-39b has a modestly eccentric orbit and is in agreement with the theoretical mass-radius relationship, whereas WASP-30b lies above it.
\end{abstract}

Key words. binaries: eclipsing - stars: individual: WASP-30 - techniques: radial velocities - stars: individual: J1219-39 stars: low-mass - brown dwarfs

\section{Introduction}

The Wide Angle Search for Planets (WASP) consortium (Pollacco et al. 2006) has been operating from La Palma, Spain, and Sutherland, South Africa. Its main goal is to find transiting extrasolar planets. With more than 80 planets discovered, this is the most successful ground-based survey for finding short-period giant planets. Amongst the many planet candidates that WASP has produced are many "false positives", which here we regard as objects of interest, that have been shown by radial-velocity

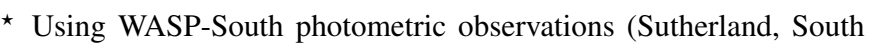
Africa) confirmed with radial velocity measurement from the CORALIE spectrograph, photometry from the EulerCam camera (both mounted on the Swiss $1.2 \mathrm{~m}$ Euler Telescope), radial velocities from the HARPS spectrograph on the ESO's $3.6 \mathrm{~m}$ Telescope (prog ID 085.C0393), and photometry from the robotic $60 \mathrm{~cm}$ TRAPPIST telescope, all located at ESO, La Silla, Chile. The data is publicly available at the CDS Strasbourg and on demand to the main author.

$\star \star$ Tables A.1-A.3 are available in electronic form at http: //www . aanda.org

$\star \star \star$ Photometry tables are only available at the CDS via anonymous ftp to cdsarc.u-strasbg.fr $(130.79 .128 .5)$ or via

http://cdsarc.u-strasbg.fr/viz-bin/qcat?J/A+A/549/A18
}

follow-up to be M dwarfs that eclipse F, G or K-dwarf companions. They are of a few Jovian radii in size and thus mimic a planetary transit signal very well. Because of the mass and low brightness of the secondaries, they remain invisible, making them convenient objects for follow-up and study using the same photometry and radial-velocity techniques that are routinely used for exoplanets. Two A+M binaries have already been presented in Bentley et al. (2009) and similar objects have been found by the OGLE survey (Udalski 2007; Pont et al. 2006) and by the HAT network (Fernandez et al. 2009).

We have made a substantial effort to characterise these lowmass eclipsing binaries (the EBLM project) in order to discover transiting brown dwarfs (such as WASP-30b, Anderson et al. 2011b) and also to complete the largely empty mass-radius diagram for stars with masses $<0.4 M_{\odot}$. These objects explore the mass distribution separating stars from planets, or serve as extended samples to the exoplanets, especially with regards to their orbital parameters, long term variability and spin-orbit angles. Our results will be published in a series of papers, of which this is the first.

A primary goal of the EBLM project is to address the M-dwarf radius problem whereby current stellar evolution 
models underestimate the radii of $\mathrm{M}$ dwarfs by at least $5 \%$ and overestimate their temperatures by a few hundred degrees (e.g. (Morales et al. 2010, 2009; López-Morales 2007) and references therein). Thus we aim to substantially increase the number of $\mathrm{M}$ dwarfs with accurate masses, radii, and metallicities using a large sample of newly discovered eclipsing binaries comprising F, G, K primaries with $\mathrm{M}$ dwarf secondaries. The masses and radii results are inferred using $\mathrm{F}, \mathrm{G}, \mathrm{K}$ atmospheric and evolution models. Although model-dependent, the analysis of bright $\mathrm{F}, \mathrm{G}, \mathrm{K}+\mathrm{M}$ dwarf eclipsing binaries promises large numbers of masses and radii of low-mass stars over the entire range of $\mathrm{M}$ dwarfs down to the hydrogen-burning limit. They will have accurate metallicity determination, and cover a wide range of activity levels. A combined analysis of the radial-velocity curve and light curve permits to deduce the masses and radii, while an accurate system metallicity can be determined from the F, G, K primary star. Furthermore, activity can be determined indirectly through knowledge of the rotation-activity relation (Morales et al. 2008) combined with $V \sin i_{\star}$ from measurements or by deduction when the systems are tidally synchronised. In addition, studying the radius anomaly while measuring the mutual inclinations, known thanks to observations of the RossiterMcLaughlin effect, we can test for the possible effect of tidal heating as outlined by Heller et al. (2010).

Holt (1893), in proposing a method to determine the rotation of stars prior to any knowledge about line broadening, predicted that when one star of a binary eclipsed the other it would first cover the advancing blue-shifted hemisphere and then the receding red-shifted part. This motion would create a colour anomaly perceived as a progressive red-shift of the primary's spectrum followed by a blue-shift, thus appearing as a symmetric radial-velocity anomaly on top of the main Doppler orbital motion of the eclipsed star's lines. This effect was first observed by Rossiter (1924) and McLaughlin (1924), though with some evidence of its presence noted earlier by Schlesinger (1910). Holt's idea was correct but only under the assumption that both stars orbit in each other's equatorial plane. In the case of a non-coplanar orbital motion the radial velocity effect is asymmetric (see e.g. Giménez 2006 or Gaudi \& Winn 2007)

Recent observations of this effect in transiting extrasolar planets (e.g. Queloz et al. 2000; Winn et al. 2005; Hébrard et al. 2008; Winn et al. 2009; Triaud et al. 2010; Moutou et al. 2011; Brown et al. 2012, and references therein) have shown that the so-called hot Jupiters, gas giant planets on orbits $<5$ days, have orbital spins on a large variety of angles with respect to the stellar spin axis, the most dramatic cases being on retrograde orbits. While it was previously thought that hot Jupiters had migrated from their formation location to their current orbits via an exchange of angular momentum with the protoplanetary disc, they are now thought to have been dynamically deflected onto highly eccentric orbits that then circularised via tidal friction. There are various ways in achieving this, such as planet-planet scattering (Rasio \& Ford 1996; Nagasawa et al. 2008; Wu \& Lithwick 2011) and Kozai resonances (Kozai 1962; Wu et al. 2007; Fabrycky \& Tremaine 2007; Naoz et al. 2011). These could be triggered by environmental effects in their original birth clusters such as fly-bys (Malmberg et al. 2007, 2011), by an additional, late, inhomogenous mass collapses in young systems (Thies et al. 2011), or during the planet formation process itself (Matsumura et al. 2010a,b).

Several patterns have emerged in the planetary spin-orbit angle data, including: a lack of aligned systems whose host stars have $T_{\text {eff }}>6250 \mathrm{~K}$ (Winn et al. 2010); a lack of inclined systems older than 2.5-3 Gyr (Triaud 2011b); and a lack of retrograde system for secondaries >5 $M_{\text {Jup }}$ (Hébrard et al. 2011; Moutou et al. 2011). To help confirm this latter trend, one could measure the Rossiter-McLaughlin effect in several heavy planets, but those are rare. It is thus easier to extend the mass range to lowmass stars, hoping to further our understanding of the planetary spin-orbit angle distribution.

The fact that hot Jupiters can be on inclined orbits raises the question about the inclinations of close binary stars. As proposed by Mazeh \& Shaham (1979), close binaries, especially those with large mass differences, might form via the same dynamical processes that have been proposed for hot Jupiters, i.e., gravitational scattering followed by tidal friction. In fact, Fabrycky \& Tremaine (2007) primarily address the formation of close binaries; the possible application to exoplanets comes later. That paper was motivated by observational results, notably presented by Tokovinin et al. (2006), showing that at least $96 \%$ of close binaries are accompanied by a tertiary component, supporting the appeal to the Kozai mechanism as described in Mazeh \& Shaham (1979). It has been argued that objects as small as $5 M_{\text {jup }}$ could be formed as stars do (Caballero et al. 2007), while objects as massive as 20 or $30 M_{\text {jup }}$ could be created by core accretion, in the fashion expected for planets (Mordasini et al. 2009). Rossiter-McLaughlin observations bridging the mass gap between planets and stars could eventually help in separating or confirming both proposed scenarii. The observation of the Rossiter-McLaughlin effect on brown dwarf mass objects can also be linked to the measure of their tidal quality factor $Q^{\prime}$ (Heller et al. 2010), which is important for studies such as those involving the early evolution of planetary orbits around such objects (Bolmont et al. 2011, 2012).

Even though attempts have been made to model the Rossiter-McLaughlin effect (e.g. Kopal 1942b; Hosokawa 1953) no systematic, quantified and unbiased survey of the projected spin-orbit angle $\beta$ in binary star systems can be found in the literature. Only isolated observations of nearly aligned systems have been reported. Kopal (1942a) mentions a possibly asymmetric Rossiter-McLaughlin effect (or rotation effect as it was then known) leading to an estimated misaligned angle of $15^{\circ}$ observed in 1923 in the Algol system, but that was presented as aligned by McLaughlin (1924). Struve (1950) writes that the rotation effect had been observed in a 100 systems without citing anyone. Slettebak (1985) is a good source of citations about this epoch. Worek (1996) and Hube \& Couch (1982) are two examples of more recent observations of the Rossiter-McLaughlin effect. The rotation effect was also used for cataclysmic variables to determine if the accreting material comes from a disc in a plane similar to the binary's orbital plane (Young \& Schneider 1980).

It has to be noted that, early on, the Rossiter-McLaughlin effect was used as a tool to measure the true rotation of a star, hence creating a bias against reporting misaligned systems. Furthermore the precision and accuracy of instrumentation, data extraction and analysing technique of that time prevented the observation of the Rossiter-McLaughlin effect for slowly rotating stars, further biasing detections of the effect towards synchronously rotating binaries, which could have tidally evolved to become aligned (Hut 1981).

In addition, the capacity to accurately model blended absorption lines of double-lined binaries (SB2) during transit has only been developed recently. Thus, most people that studied binaries chose not to observe during eclipses. Modelling eclipsing SB2 has recently been developed in Albrecht et al. (2007), and used by Albrecht et al. (2009) for the case of DI Herculis, explaining its previously abnormal apsidal motion: both stars orbit above 
A. H. M. J. Triaud et al.: EBLM project. I.

each other's poles. These measurements are being followed by a systematic and quantified survey of spin-orbit measurements for SB2s of hot stars with similar masses (the BANANA project, Albrecht et al. 2011a, who also compile many older results). Another contemporary result is presented in an asteroseismologic paper by Desmet et al. (2010).

We circumvent the blended-line problem altogether by only observing the WASP candidates that turned out to be single-line binaries (SB1) while searching for extrasolar planets. Low-mass M dwarfs and brown dwarfs have a size similar to gas giants and appear to a first approximation like a planet: a dark spot moving over the disc of their primary. Thus, the low-mass eclipsing binaries found by transiting planet surveys provide a good sample to extend the work carried out on planets and provide a largely unbiased, quantified survey of spin-orbit angles for F, G or $\mathrm{K}+\mathrm{M}$ binaries, complementary to the BANANA project. The differences between our primaries will also allow us to probe the way tides propagate in convective or radiative stars (Zahn 1977). In stellar parameters and data treatment, our systems resemble the aligned pair Kepler-16 A \& B (Doyle et al. 2011; Winn et al. 2011), but with shorter periods.

In this we first present our observations of WASP-30 and J1219-39 (1SWASPJ121921.03-395125.6, TYC 7760-484-1), then describe our models and how they were adjusted to fit the data, and how the error bars were estimated. We will then move to a discussion of the results.

\section{Observations}

The discovery of WASP-30 was announced in Anderson et al. (2011b). This is a transiting - or eclipsing - $61 M_{\text {Jup }}$ brown dwarf on a 4.16-day orbit. In our analysis we have used the data published in Anderson et al. (2011b) as well as new observations. The full sample comprises photometric observations from three facilities: the WASP-South photometry (four datasets totalling 17528 independent measurements) and the Gunn $r^{\prime}$ Euler photometry (one set of 250 points) were presented in Anderson et al. (2011b). In addition we present 571 new photometric observations obtained in the $I+z$ band using the TRAPPIST telescope, covering the transit of 2010 October 15 . We also gathered radial-velocity data: 32 spectra were observed using CORALIE (mounted on the Swiss $1.2 \mathrm{~m}$ Euler Telescope) of which 16 have been published by Anderson et al. (2011b). We also acquired 37 measurements using HARPS-South on the ESO $3.6 \mathrm{~m} .8$ CORALIE and 16 HARPS measurements were obtained during the transits of 2010 October 15 and 2010 September 20, thus recording the Rossiter-McLaughlin effect.

$\mathrm{J} 1219-39$ is located at $\alpha=12^{\mathrm{h}} 19^{\prime} 21.03^{\prime \prime}$ and $\delta=$ $-39^{\circ} 51^{\prime} 25.6^{\prime \prime}$. Its name is a short version of its WASP catalogue entry. WASP-South observed a total of 22032 points in four series of photometric measurements obtained between 2006 May 04 and 2008 May 28. The automated Hunter algorithm (Collier Cameron et al. 2007b) found a periodic signal with period 6.76 days. This period was confirmed with 20 out-oftransit radial-velocity measurements obtained with CORALIE between 2008 August 03 and 2011 April 17. We also acquired an additional 54 measurements by observing nights during which three primary eclipses occurred (on 2010 May 13, 2010 July 13 and 2011 April 16). Several spectra were affected by bad weather conditions. Points with error bars above $20 \mathrm{~m} \mathrm{~s}^{-1}$ were thus removed, leaving $61 \mathrm{RV}$ points with an average error of $9.9 \mathrm{~m} \mathrm{~s}^{-1}$. Of these, 19 were taken during the Rossiter-McLaughlin effect. Because the aim of this paper is not about characterising the radius of this object but about orbital parameters, the WASP
Table 1. Stellar parameters and abundances of our two primaries from spectral line analysis.

\begin{tabular}{|c|c|c|}
\hline & WASP-30A & J1219-39A \\
\hline 1SWASP & $\mathrm{J} 235338.03-100705.1$ & J121921.03-395125.6 \\
\hline 2MASS & J23533805-1007049 & J12192104-3951256 \\
\hline TYC & 5834-95-1 & 7760-484-1 \\
\hline & F8V & K0V \\
\hline$\alpha$ & $23^{\mathrm{h}} 53^{\prime} 38.05^{\prime \prime}$ & $12^{\mathrm{h}} 19^{\prime} 21.03^{\prime \prime}$ \\
\hline$\delta$ & $-10^{\circ} 07^{\prime} 05.1^{\prime \prime}$ & $-39^{\circ} 51^{\prime} 25.6^{\prime \prime}$ \\
\hline$V$ mag & 11.9 & 10.3 \\
\hline$T_{\text {eff }}(\mathrm{K})$ & $6190 \pm 50$ & $5400 \pm 80$ \\
\hline $\log g$ & $4.18 \pm 0.08$ & $4.5 \pm 0.1$ \\
\hline$\xi_{\mathrm{t}}\left(\mathrm{km} \mathrm{s}^{-1}\right)$ & $1.1 \pm 0.1$ & $1.0 \pm 0.1$ \\
\hline$v_{\text {mac }}\left(\mathrm{km} \mathrm{s}^{-1}\right)$ & $3.8 \pm 0.3$ & $1.4 \pm 0.3$ \\
\hline$v \sin i_{\star}\left(\mathrm{km} \mathrm{s}^{-1}\right)$ & $12.1 \pm 0.5$ & $4.5 \pm 0.4$ \\
\hline$[\mathrm{Fe} / \mathrm{H}]$ & $0.07 \pm 0.08$ & $-0.23 \pm 0.08$ \\
\hline$[\mathrm{Na} / \mathrm{H}]$ & $0.09 \pm 0.04$ & $-0.15 \pm 0.05$ \\
\hline$[\mathrm{Ca} / \mathrm{H}]$ & $0.11 \pm 0.12$ & $-0.07 \pm 0.11$ \\
\hline$[\mathrm{Ti} / \mathrm{H}]$ & $0.02 \pm 0.10$ & $-0.08 \pm 0.06$ \\
\hline$[\mathrm{Cr} / \mathrm{H}]$ & $0.11 \pm 0.10$ & $-0.17 \pm 0.0$ \\
\hline$[\mathrm{Ni} / \mathrm{H}]$ & $0.04 \pm 0.08$ & $-0.19 \pm 0.08$ \\
\hline$[\mathrm{Mg} / \mathrm{H}]$ & - & $-0.10 \pm 0.05$ \\
\hline$[\mathrm{Al} / \mathrm{H}]$ & - & $-0.17 \pm 0.08$ \\
\hline$[\mathrm{Si} / \mathrm{H}]$ & - & $-0.13 \pm 0.07$ \\
\hline$[\mathrm{Sc} / \mathrm{H}]$ & - & $-0.18 \pm 0.06$ \\
\hline$[\mathrm{V} / \mathrm{H}]$ & - & $-0.01 \pm 0.09$ \\
\hline$[\mathrm{Mn} / \mathrm{H}]$ & - & $-0.11 \pm 0.13$ \\
\hline$[\mathrm{Co} / \mathrm{H}]$ & - & $-0.11 \pm 0.06$ \\
\hline $\log A(\mathrm{Li})$ & $2.98 \pm 0.04$ & $<0.15$ \\
\hline
\end{tabular}

from the Torres et al. (2010) relation
$M_{\star}\left(M_{\odot}\right)$
$1.28 \pm 0.09$
$0.89 \pm 0.06$
$R_{\star}\left(R_{\odot}\right)$
$1.51 \pm 0.17$
$0.87 \pm 0.11$

facilities used $\mathcal{E}$ number of observations used in the analysis

$\begin{array}{lcc}\text { WASP-South }[\mathrm{V}+\mathrm{R}] & 17528 & 22032 \\ \text { TRAPPIST }[\mathrm{I}+z] & 571 & - \\ \text { EulerCam }\left[r^{\prime}\right] & 250 & - \\ \text { CORALIE } & 32 & 61 \\ \text { HARPS } & 37 & -\end{array}$

Notes. Spectral Type estimated from $T_{\text {eff }}$ using the Table in Gray (2008).

photometry is the only photometry we will use, which is sufficient to help in constraining the Rossiter-McLaughlin effect. This however does not prevent us from using the fact that the object eclipses to help get its mass and infer an estimate of its radius.

Additional details are located in the observational journal in the appendices, and a summary is displayed in Table 1 .

\section{Data treatment}

\subsection{The WASP-South photometry}

We used standard aperture photometry as described in Sect. 4.3 of Pollacco et al. (2006) where a 3.5-pixel aperture around the source is used (with the source position taken from a catalogue). Sky subtraction comes from an annulus (with radii of 13 to 17 pixels). Regions around catalogued stars and cosmic rays are removed from that calculation. The pixel scale is 13.7" per pixel. To maximise photons, we observed in white light, only with a 

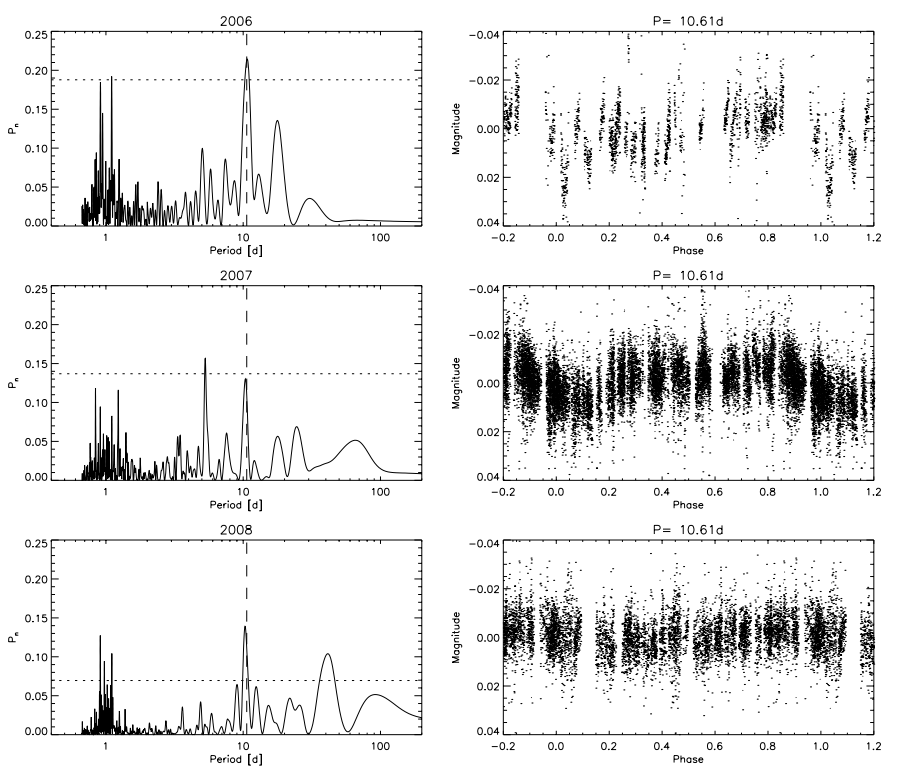

Fig. 1. Left panels: periodograms for the WASP data from the different observing seasons for J1219-39. Horizontal lines indicate false alarm probability levels FAP $=0.01$ and vertical lines show our assumed rotational modulation period of $P=10.6 \mathrm{~d}$. The year of observation in given in the title. Right panels: lightcurves folded on the period $P=10.61 \mathrm{~d}$.

cut-off filter in the far red in order to reduce effects from fringing. This is a large bandpass approximating to $\mathrm{V}+\mathrm{R}$.

We used the sine-wave fitting method described in Maxted et al. (2011) to search for any periodicity in the WASP lightcurves owing to the rotation of the stars and caused by magnetic activity, i.e., star spots. Spot-induced variability is not expected to be coherent on long timescales as a consequence of the finite lifetime of star-spots and of differential rotation in the photosphere so we analysed each season of WASP data separately. We first subtracted a simple transit model from the lightcurve. We then calculated periodograms over 4096 uniformly spaced frequencies from 0 to 1.5 cycles/day (Fig. 1). The false-alarm probability levels shown in these figures are calculated using a boot-strap Monte Carlo method also described in Maxted et al. (2011).

For WASP-30 we analysed WASP lightcurves from 3 different seasons with several thousand observations over about 100 days. These lightcurves show no significant periodic out-oftransit variability. We examined the distribution of amplitudes for the most significant frequency in each Monte Carlo trial and used these results to estimate a 95\% upper confidence limit of 0.8 milli-magnitude for the amplitude of any periodic signal in these lightcurves.

The results for our periodogram analysis of J1219-39 are shown in Table 2. Our interpretation is that we do detect rotational modulation of J1219-39 and that in the 2007 data the pattern of spots results in the strongest signal being seen at $P_{\text {rot }} / 2$, where $P_{\text {rot }}$ is the rotation period of the star at the latitude of the star spots. This leads to $P_{\text {rot }}=10.61 \pm 0.05 \mathrm{~d}$ calculated from the unweighted mean and standard error on the mean from the three seasons. The periodograms for these data and the lightcurves folded on this value of $P_{\text {rot }}$ are shown in Fig. 1.
Table 2. Frequency analysis for J1219-39.

\begin{tabular}{ccccc}
\hline \hline Year & $N$ & $P($ day $)$ & Amp $(\mathrm{mmag})$ & FAP \\
\hline 2006 & 1855 & 10.710 & 6 & 0.027 \\
2007 & 12104 & 5.292 & 5 & 0.004 \\
2008 & 6019 & 10.300 & 3 & 0.001 \\
\hline
\end{tabular}

Notes. $N$ is the number of observations, $P$ is the period corresponding to the strongest peak in the periodogram, Amp is the amplitude of the bestfit sine wave in milli-magnitudes and FAP is the false-alarm probability.

\subsection{The TRAPPIST I + z-band photometry}

A complete transit of WASP-30 was observed with the robotic $60 \mathrm{~cm}$ telescope TRAPPIST ${ }^{1}$ (Gillon et al. 2011; Jehin et al. 2011). Located at La Silla ESO observatory (Chile), TRAPPIST is equipped with a $2 \mathrm{~K} \times 2 \mathrm{~K}$ Fairchild $3041 \mathrm{CCD}$ camera that has a $22^{\prime} \times 22^{\prime}$ field of view (pixel scale $=0.64^{\prime \prime} /$ pixel). The transit of WASP-30 was observed on the night of 2010 October 15 . The sky conditions were clear. We used the $1 \times 2 \mathrm{MHz}$ read-out mode with $1 \times 1$ binning, resulting in a typical read-out + overhead time and read noise of $8.2 \mathrm{~s}$ and $13.5 e^{-}$, respectively. The integration time was 30 s for the entire night. We observed through a special $I+z$ filter that has a transmittance of zero below $700 \mathrm{~nm}$, and $>90 \%$ from $750 \mathrm{~nm}$ to beyond $1100 \mathrm{~nm}$. The telescope was defocused to average pixel-to-pixel sensitivity variations and to optimise the duty cycle, resulting in a typical full width at halfmaximum of the stellar images of $\sim 6$ pixels $\left(\sim 3.8^{\prime \prime}\right)$. The positions of the stars on the chip were maintained to within a few pixels over the course of two timeseries, separated by a meridian flip, thanks to the "software guiding" system that regularly derives an astrometric solution from the most recently acquired image and sends pointing corrections to the mount if needed. After a standard pre-reduction (bias, dark, flat field), the stellar fluxes were extracted from the images using the IRAF/DAOPHOT aperture photometry software (Stetson 1987). Several sets of reduction parameters were tested, and we kept the one giving the most precise photometry for the stars of brightness similar to WASP-30. After a careful selection of reference stars, differential photometry was obtained (Gillon et al. 2012). The data are shown in Fig. 2. Because of a meridian flip inside the transit the photometry was analysed as two independent timeseries.

\subsection{The radial velocity data}

The spectroscopic data were reduced using the online Data Reduction Software (DRS) for the HARPS instrument. The radial-velocity information was obtained by removing the instrumental blaze function and cross-correlating each spectrum with a mask. This correlation was compared with the Th-Ar spectrum used as a wavelength-calibration reference (see Baranne et al. 1996; and Pepe et al. 2002, for details). The DRS has been shown to achieve remarkable precision (Mayor et al. 2009) thanks to a revision of the reference lines for thorium and argon by Lovis \& Pepe (2007). A similar software package was used to prepare the CORALIE data. A resolving power $R=110000$ for HARPS provided a cross-correlation function (CCF) binned in $0.25 \mathrm{~km} \mathrm{~s}^{-1}$ increments, while for the CORALIE data, with a lower resolution of 50000 , we used $0.5 \mathrm{~km} \mathrm{~s}^{-1}$. The CCF window was adapted to be three times the size of the full width at half maximum (FWHM) of the CCF.

1 http://arachnos.astro.ulg.ac.be/Sci/Trappist 

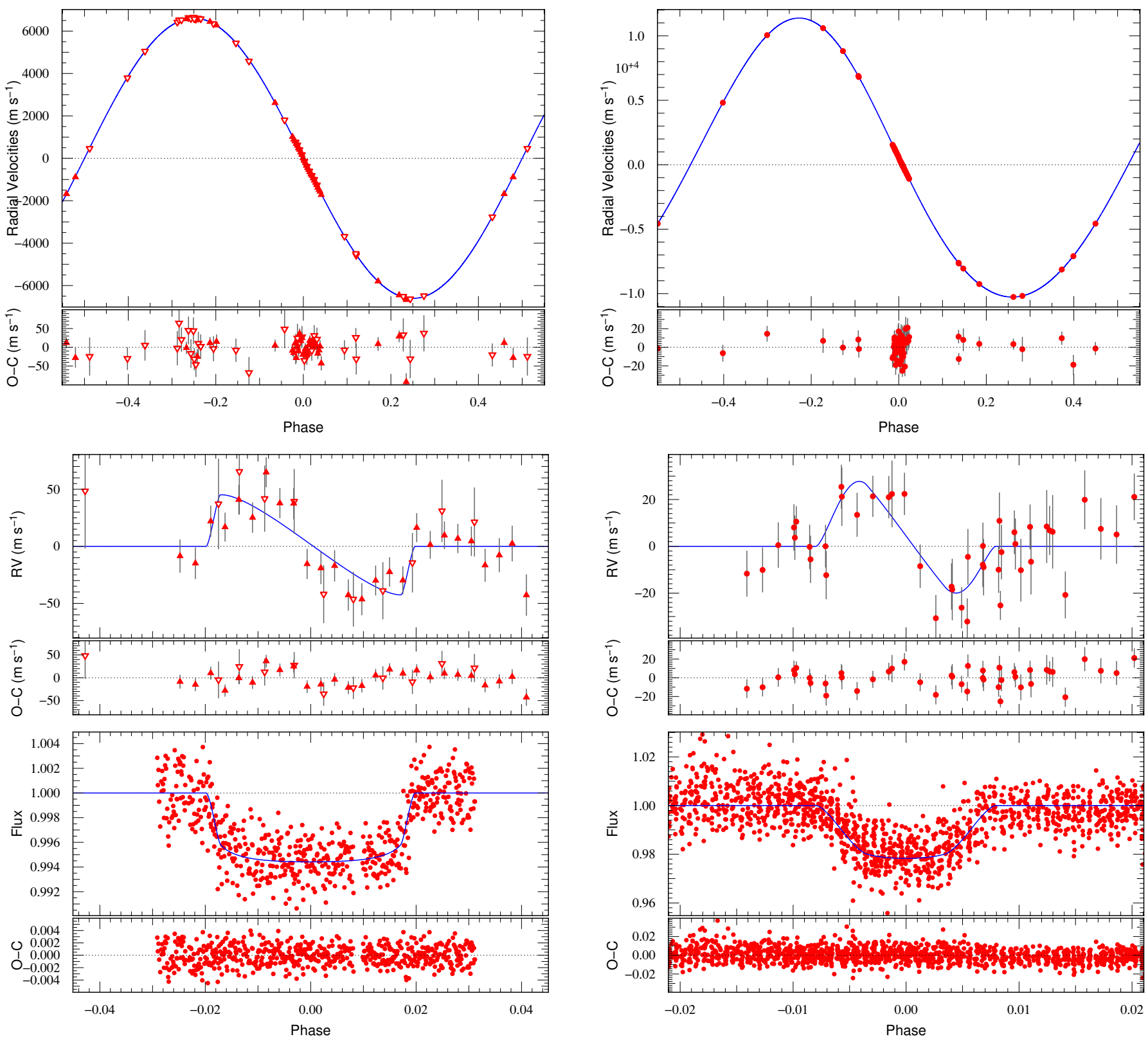

Fig. 2. Top: radial velocities on WASP-30 plotted with a circular Keplerian model and their residuals. CORALIE data is shown as inverted, empty, triangles. HARPS is show as upright triangles. Middle: zoom on the Rossiter-McLaughlin effect. Bottom: TRAPPIST $I+z$ photometry and model over-plotted. The interruption of the observations is due to a telescope meridian flip.

$1 \sigma$ error bars on individual data points were estimated from photon noise alone. HARPS is stable in the long term to within $1 \mathrm{~m} \mathrm{~s}^{-1}$ and CORALIE to better than $5 \mathrm{~m} \mathrm{~s}^{-1}$. These are smaller than our individual error bars, and thus were not taken into account.

As in the initial discovery paper, for WASP-30, a G2 mask was used. In the case of J1219-39 we used a K5 mask to extract the radial-velocity information.

Several points were removed from the analysis. For WASP-30, we excluded a mistakenly obtained series of 13 short CORALIE spectra taken during bad weather; the error bars are all above $>100 \mathrm{~m} \mathrm{~s}^{-1}$ when no other data has errors $>50 \mathrm{~m} \mathrm{~s}^{-1}$. On J1219-39b bad weather also affected observations, notably

Fig. 3. Top: CORALIE radial velocities on J1219-39 plotted with an eccentric Keplerian model and their residuals. Middle: zoom on the Rossiter-McLaughlin effect. Bottom: WASP photometry and model over-plotted.

during one of the Rossiter-McLaughlin sequences. All measurements with error bars $>20 \mathrm{~m} \mathrm{~s}^{-1}$ have been removed from the analysis as they show a clear jump in precision from other measurements. All the rejected radial velocities are nevertheless presented in the journal of observations, in the appendices, and are clearly indicated.

\section{Spectral analysis}

The analysis was performed following the methods detailed in Gillon et al. (2009b) with standard pipeline reduction products used in the analysis. The $H_{\alpha}$ line was used to determine the effective temperature $\left(T_{\text {eff }}\right)$. The surface gravity $(\log g)$ was determined from the $\mathrm{Ca}$ I lines at $6162 \AA$ and $6439 \AA$ (Bruntt et al. 2010 b), along with the $\mathrm{Na}$ I $\mathrm{D}$ lines. Additional $T_{\text {eff }}$ and $\log g$ 
diagnostics were performed using the Fe lines. An ionisation balance between $\mathrm{Fe} \mathrm{I}$ and $\mathrm{Fe}$ II was required, along with a null dependence of the abundance on either equivalent width or excitation potential (Bruntt et al. 2008). The parameters obtained from the analysis are listed in Table 1. The elemental abundances were determined from equivalent width measurements of several clean and unblended lines. A value for microturbulence $\left(\xi_{\mathrm{t}}\right)$ was determined from Fe I using the method of Magain (1984). The quoted error estimates include that given by the uncertainties in $T_{\text {eff }}, \log g$ and $\xi_{\mathrm{t}}$, as well as the scatter due to measurement and atomic data uncertainties.

A total of 37 individual HARPS spectra of WASP-30A were co-added to produce a single spectrum with a typical signal-tonoise ratio $(\mathrm{S} / \mathrm{N})$ of around 120:1. Interstellar Na D lines are present in the spectra with an equivalent widths of $\sim 0.16 \AA$, indicating an extinction of $E(B-V)=0.05$ using the calibration of Munari \& Zwitter (1997). The projected stellar rotation velocity $\left(v \sin i_{\star}\right)$ was determined by fitting the profiles of several unblended $\mathrm{Fe}$ I lines. A value for macroturbulence $\left(v_{\mathrm{mac}}\right)$ of $3.8 \pm 0.3 \mathrm{~km} \mathrm{~s}^{-1}$ was assumed, based on the calibration by Bruntt et al. (2010a). An instrumental FWHM of $0.07 \pm 0.01 \AA$ was determined from the telluric lines around $6300 \AA$. A best fitting value of $v \sin i_{\star}=12.1 \pm 0.5 \mathrm{~km} \mathrm{~s}^{-1}$ was obtained.

The lithium abundance would imply an age no more than $\sim 0.5$ Gyr (Sestito \& Randich 2005) but stars with $T_{\text {eff }}$ similar to WASP-30A in M67 (5 Gyr old) have shown similar abundances (Fig. 6 in Sestito \& Randich 2005). Those results are in agreement with the analysis of 16 co-added CORALIE spectra that was published by Anderson et al. (2011b).

A similar analysis was conducted on J1219-39A. Individual spectra were combined to a single spectrum of $\mathrm{S} / \mathrm{N}$ typically 150:1. Using a macroturbulence $v_{\mathrm{mac}}=1.4 \pm 0.3 \mathrm{~km} \mathrm{~s}^{-1}$ (Bruntt et al. 2010a) we obtain a $v \sin i_{\star}=4.5 \pm 0.4 \mathrm{~km} \mathrm{~s}^{-1}$. Using the macroturbulence calibration from Valenti \& Fischer (2005), we find $v_{\mathrm{mac}}=3.4 \pm 0.3 \mathrm{~km} \mathrm{~s}^{-1}$, then we can infer $v \sin i_{\star}=3.3 \pm 0.4 \mathrm{~km} \mathrm{~s}^{-1}$. We will see later both those values are in contradiction with the $V \sin i_{\star}$, directly measured from the Rossiter-McLaughlin effect with the latter value being the closest. Here no Lithium can be detected for an equivalent width $<1 \mathrm{~mA}$. The cores of the $\mathrm{Ca} \mathrm{H} \& \mathrm{~K}$ lines show some emission indicative of stellar activity and in agreement with the detection of spot-induced variability (Sect. 3.1).

We will make a distinction in this paper between $v \sin i_{\star}$, the projected rotational velocity of the star computed by estimating the stellar line broadening, and the $V \sin i_{\star}$, which is the same physical quantity, but obtained directly from the amplitude of the Rossiter-McLaughlin effect. We also distinguish $i_{\star}$ the inclination of the stellar spin, from $i$, the inclination of the orbital spin of the companion.

\section{Model adjustment}

Simple Keplerian models were fitted to the radial-velocity data simultaneously with transit models from Mandel \& Agol (2002) fitted to the photometry, and a Rossiter-McLaughlin model by Giménez (2006) fitted to RV points falling within transit/eclipse. We used a quadratic limb-darkening law, and obtained parameters from Claret (2004) to apply to the photometry. For the Rossiter-McLaughlin effect, we applied parameters derived by Claret for HARPS (Triaud et al. 2009). A Markov chain Monte Carlo (MCMC) was used to compare the data and the models and explore parameter space to find the most likely model with robust confidence intervals on each parameter. Having only one set of parameters for all datasets ensures the parameter distributions are consistent with all of the data. The algorithm is widely described in several planet-discovery papers from the WASP consortium (e.g. Collier Cameron et al. 2007a; Gillon et al. 2009a; Anderson et al. 2011a; Triaud 2011a). The same method is used here with one important difference:

While adjusting for planets, many authors have used the property that the mass of the planet is much less than the mass of the star $\left(M_{2} \ll M_{\star}\right)$. This assumption is made in several places: in calculating the mass ratio from the mass function, the radii from the scaled radii $R_{2} / a$ and $R_{\star} / a$, and in calculating the stellar density $\rho_{\star}$. This last parameter is important since, being more precise than the traditional $\log g$, it is widely used to infer stellar parameters (Sozzetti et al. 2007).

Three methods are often used in the literature involving MCMC fitting algorithms that use the mean stellar density to obtain $M_{\star}$. One can fit the transit to obtain $\rho_{\star}$, use it to infer the stellar mass by interpolating of stellar tracks, and then insert the new $M_{\star}$ back into a chain, as employed by Hebb et al. (2009). Alternatively Enoch et al. (2010) have devised an empirical relation based on the Torres relation (Torres et al. 2010) which also delivers $M_{\star}$. Finally one can mix both previous methods and estimate $M_{\star}$ at every step of an MCMC by using $\rho_{\star}$ to interpolate within theoretical stellar tracks as shown in Triaud (2011a) and in Gillon et al. (2012). $\rho_{\star}$ is defined, from Kepler's law as:

$$
\frac{M_{\star}}{R_{\star}^{3}}=\frac{4 \pi^{2}}{G P^{2}}\left(\frac{a}{R_{\star}}\right)^{3}-\frac{M_{2}}{R_{\star}^{3}} .
$$

In our case, the second term can no longer be considered null. In order to still be able to use the more precise $\rho_{\star}$ over $\log g$, we proceeded as follow: for every step in the MCMC we use the transit geometry to estimate the secondary's orbital inclination $i$. Then the mass function (Hilditch 2001)

$f(m)=\left(1-e^{2}\right)^{3 / 2} \frac{P K^{3}}{2 \pi G}$

is estimated. It can also be written as

$f(m)=\frac{\left(M_{2} \sin i\right)^{3}}{\left(M_{\star}+M_{2}\right)^{2}}$.

Equating both, we can numerically solve for $M_{2}$ assuming $M_{\star}$ (for example at the start of the chain, from the Torres relation). The orbital separation can then be estimated, and, having $R_{\star} / a$ from the transit signal, we obtain $R_{\star}$. We thus have gathered first estimates of all quantities necessary to compute $\rho_{\star}$. This value is then combined with $[\mathrm{Fe} / \mathrm{H}]$ and $T_{\text {eff }}$ to give $M_{\star}$ from interpolating within stellar evolution tracks. $M_{\star}$ can then be used to re-estimate $M_{2}$ and $R_{\star}$, via the same path as outlined above, from which $R_{2}$ is also determined, using the transit depth. This is repeated for each of the 2000000 steps of our MCMCs.

We use a new version of the Geneva stellar evolution tracks, described in Mowlavi et al. (2012) and for the moment make the assumption that the primary is located on the main sequence. Because the steps falling outside the tracks are rejected, and provided the star is indeed on the main sequence, fitting using the tracks has the advantage of only allowing physically possible stars to be used in the MCMC. It has also the capacity to check and refine stellar parameters derived from spectral analysis, especially with respect to the lower boundary composed by the zero age main sequence.

Our Markov chains use the following jump parameters: $D$, the photometric transit/eclipse depth, $W$, its width, $b$, the impact 
A. H. M. J. Triaud et al.: EBLM project. I.

parameter, $K$, the semi-amplitude of the radial velocity signal, $P$, the period, $T_{0}$, the transit mid-time point at the barycentre of the data (RV and photometric, weighted by their respective signal to noise). We also have two pairs of parameters: $\sqrt{e} \cos \omega$ $\& \sqrt{e} \cos \omega$ and $\sqrt{V \sin i_{\star}} \sin \beta \& \sqrt{V \sin i_{\star}} \cos \beta$ where $e$ is the eccentricity, $\omega$ is the angle of periastron, $V \sin i_{\star}$ is the rotation velocity of the star and $\beta$ is the projected spin-orbit angle. Those parameters are combined together to avoid inserting a bias in the determination of $e$ and $V \sin i_{\star}$ (see Ford 2006; Triaud et al. 2011, for details). $[\mathrm{Fe} / \mathrm{H}]$ and $\mathrm{T}_{\text {eff }}$ are drawn randomly from a normal distribution taken from our spectral analysis. Normalisation factors for the photometry, and $\gamma$ velocities for each RV datasets, are not floating, but computed. For WASP-30, the RV data was cut into four datasets: the CORALIE data on the orbit, the CORALIE data during RM effect plus one measurement the night before and after, the HARPS data on the orbit, the HARPS RM effect plus one point the night before, and one the night after. Several chains are run to ensure, first, that convergence is achieved, but also to test the effects of different priors.

From the jump parameters a number of physical parameters, such as the masses and radii of both objects, can be computed. Useful, assumption-free parameters are also available, such as the secondary's surface gravity $\log g_{2}$, as noted in Southworth et al. (2004).

Results are taken as the modes of the posterior probability distributions. Errors for each parameter are obtained around the mode using the marginalised distribution and taking the 1-, 2and $3-\sigma$ confidence regions.

\section{Results}

\subsection{WASP-30b}

Several chains were run on WASP-30b, exploring the effect that various priors could have on the end results. Overall the fit between the data and the models is good. In order to get a $\chi_{\text {reduced }}^{2}$ close to 1 , an extra contribution of $5 \mathrm{~m} \mathrm{~s}^{-1}$ was added quadratically to the errors of the radial-velocity data. This stems mostly from one point in the HARPS data as well as from some high-cadence noise during the Rossiter-McLaughlin sequence. Nevertheless, we achieve a dispersion after the models are subtracted of $26.8 \mathrm{~m} \mathrm{~s}^{-1}$ for a $\chi_{\text {reduced }}^{2}=1.47 \pm 0.21$.

The main difference between this analysis and that presented by Anderson et al. (2011b) is a slight change in mass and radius of WASP-30b, arising from different choices for the estimation of the primary's parameters. Anderson et al. (2011b) used a Main-Sequence prior, which forced the photometric fit to be compatible with a smaller and less massive star. Upon relaxing the prior, those authors obtain a solution for the primary that is close to ours. The Main-Sequence prior is also the reason for a slightly different transit duration between the discovery paper and the current solution. It forced a solution through the initial data which is no longer compatible with the addition of the TRAPPIST light curve. The data in the discovery paper only had WASP photometry and an Euler light curve that was imprecise during ingress. A small additional contribution comes from no longer making the planet approximation.

Using priors on the values of $M_{\star}$ and $R_{\star}$ obtained using the Torres relation does not affect the result. Fits not using them are thus preferable as they constitute an independent measurement. WASP-30A is found to be a $1.25 \pm 0.03 M_{\odot}, 1.39 \pm 0.03 R_{\odot}$ star at the end of its main-sequence lifetime. The values for $T_{\text {eff }}$ and $[\mathrm{Fe} / \mathrm{H}]$ from the output of the MCMC (Table 3) are entirely
Table 3. Floating and computed parameters found for our two systems WASP-30A\&b and J1219-39A\&b.

\begin{tabular}{lcc}
\hline \hline Parameters (units) & WASP-30 & $\mathrm{J} 1219-39$ \\
\hline jump parameters & & \\
$P$ (days) & $4.156739_{-(10)}^{+(12)}$ & $6.7600098_{-(22)}^{+(34)}$ \\
$T_{0}$ (BJD-2 450 000) & $5443.06046_{-(33)}^{+(43)}$ & $5187.72676_{-(41)}^{+(29)}$ \\
$D$ & $0.00494_{-(13)}^{+(11)}$ & $0.02088_{-(69)}^{+(89)}$ \\
$W($ days) & $0.1644_{-(09)}^{+(13)}$ & $0.1040_{-(20)}^{+(20)}$ \\
$b\left(\mathrm{R}_{\star}\right)$ & $0.10_{-(0.10)}^{+(0.12)}$ & $0.733_{-(31)}^{+(2)}$ \\
$K\left(\mathrm{~m} \mathrm{~s}^{-1}\right)$ & $6606.7_{-(5.3)}^{+(4.7)}$ & $10822.2_{-(3.1)}^{+(2.8)}$ \\
$\sqrt{V \sin i_{\star}} \cos \beta$ & $3.40_{-(0.24)}^{+(0.12)}$ & $1.61_{-(0.11)}^{+(0.11)}$ \\
$\sqrt{V \sin i_{\star}} \sin \beta$ & $0.5_{-(1.6)}^{+(1.1)}$ & $0.13_{-(0.115)}^{+(0.15)}$ \\
$\sqrt{e} \cos \omega$ & 0 (fixed) & $0.21932_{-(48)}^{+(57)}$ \\
$\sqrt{e} \sin \omega$ & 0 (fixed) & $0.08537_{-(89)}^{+(85)}$
\end{tabular}

derived parameters

\begin{tabular}{|c|c|c|}
\hline$f(m)\left(M_{\odot}\right)$ & $0.00012418_{-(29)}^{+(28)}$ & $0.000883709_{-(71)}^{+(71)}$ \\
\hline$R_{2} / R_{\star}$ & $0.0704_{-(10)}^{+(07)}$ & $0.1446_{-(25)}^{+(29)}$ \\
\hline$R_{\star} / a$ & $0.1164_{-(12)}^{+(25)}$ & $0.0561_{-(23)}^{+(18)}$ \\
\hline$\rho_{\star}\left(\rho_{\odot}\right)$ & $0.466_{-(29)}^{+(16)}$ & $1.50_{-(0.17)}^{+(0.17)}$ \\
\hline$R_{\star}\left(R_{\odot}\right)$ & $1.389_{(25)}^{(33)}$ & $0.811_{-(24)}^{+(38)}$ \\
\hline$M_{\star}\left(M_{\odot}\right)$ & $1.249_{-(36)}^{+(32)}$ & $0.826_{-(29)}^{+(32)}$ \\
\hline $\log g_{\star}(\mathrm{cgs})$ & $4.250_{-(18)}^{+(09)}$ & $4.523_{-(26)}^{+(39)}$ \\
\hline$R_{2} / a$ & $0.00821_{-(18)}^{+(19)}$ & $0.00817_{-(48)}^{+(33)}$ \\
\hline$R_{2}\left(\mathrm{R}_{\mathrm{Jup}}\right)$ & $0.951_{-(24)}^{+(28)}$ & $1.142_{-(49)}^{+(69)}$ \\
\hline$M_{2}\left(M_{\mathrm{Jup}}\right)$ & $62.5_{-(1.2)}^{+(1.2)}$ & $95.4_{-(2.5)}^{+(1.9)}$ \\
\hline $\log g_{2}(\mathrm{cgs})$ & $5.234_{-(22)}^{+(19)}$ & $5.245_{-(42)}^{+(47)}$ \\
\hline$a(\mathrm{AU})$ & $0.05534_{-(51)}^{+(47)}$ & $0.06798_{-(77)}^{+(83)}$ \\
\hline$i\left(^{\circ}\right)$ & $89.43_{-(0.93)}^{+(0.51)}$ & $87.61_{-(0.18)}^{+(0.17)}$ \\
\hline$\beta\left(^{\circ}\right)$ & $7_{-(27)}^{+(19)}$ & $4.1_{-(5.3)}^{+(4.8)}$ \\
\hline$e$ & $<0.0044$ & $0.05539_{-(22)}^{+(23)}$ \\
\hline$\omega\left(^{\circ}\right)$ & - & $21.26_{-(0.23)}^{+(0.21)}$ \\
\hline$|\dot{\gamma}|\left(\mathrm{m} \mathrm{s}^{-1} \mathrm{yr}^{-1}\right)$ & $<53$ & $<10$ \\
\hline$V \sin i_{\star}\left(\mathrm{km} \mathrm{s}^{-1}\right)$ & $12.1_{-(0.5)}^{+(0.4)}$ & $2.61_{-(35)}^{+(42)}$ \\
\hline$T_{\text {eff }}(\mathrm{K})$ & $6202_{-(51)}^{+(42)}$ & $5412_{-(65)}^{+(81)}$ \\
\hline$[\mathrm{Fe} / \mathrm{H}]$ & $0.083_{-(50)}^{+(169)}$ & $-0.209_{-(75)}^{+(70)}$ \\
\hline Age (Gyr) & $3.4_{-(0.5)}^{+(0.3)}$ & 6-12 \\
\hline$\gamma_{\text {coralie }}\left(\mathrm{km} \mathrm{s}^{-1}\right)$ & $7.9307_{-(17)}^{+(22)}$ & $33.7971_{-(15)}^{+(16)}$ \\
\hline$\gamma_{\text {harps }}\left(\mathrm{km} \mathrm{s}^{-1}\right)$ & $7.87472_{-(31)}^{+(36)}$ & $-^{-(11)}$ \\
\hline
\end{tabular}

Notes. For clarity only the last two digits of the $1 \sigma$ errors are shown.

compatible with those presented in Table 1, meaning that we suffer little bias due to proximity of the star to the terminalage main sequence. Thus, using these stellar parameters we find that WASP-30b is a $62.5 \pm 1.2 M_{\text {Jup }}$ brown dwarf with a radius of $0.95 \pm 0.03 R_{\text {Jup }}$. No eccentricity is detected. We can place a $95 \%$-confidence upper limit of $e<0.0044$. No additional 

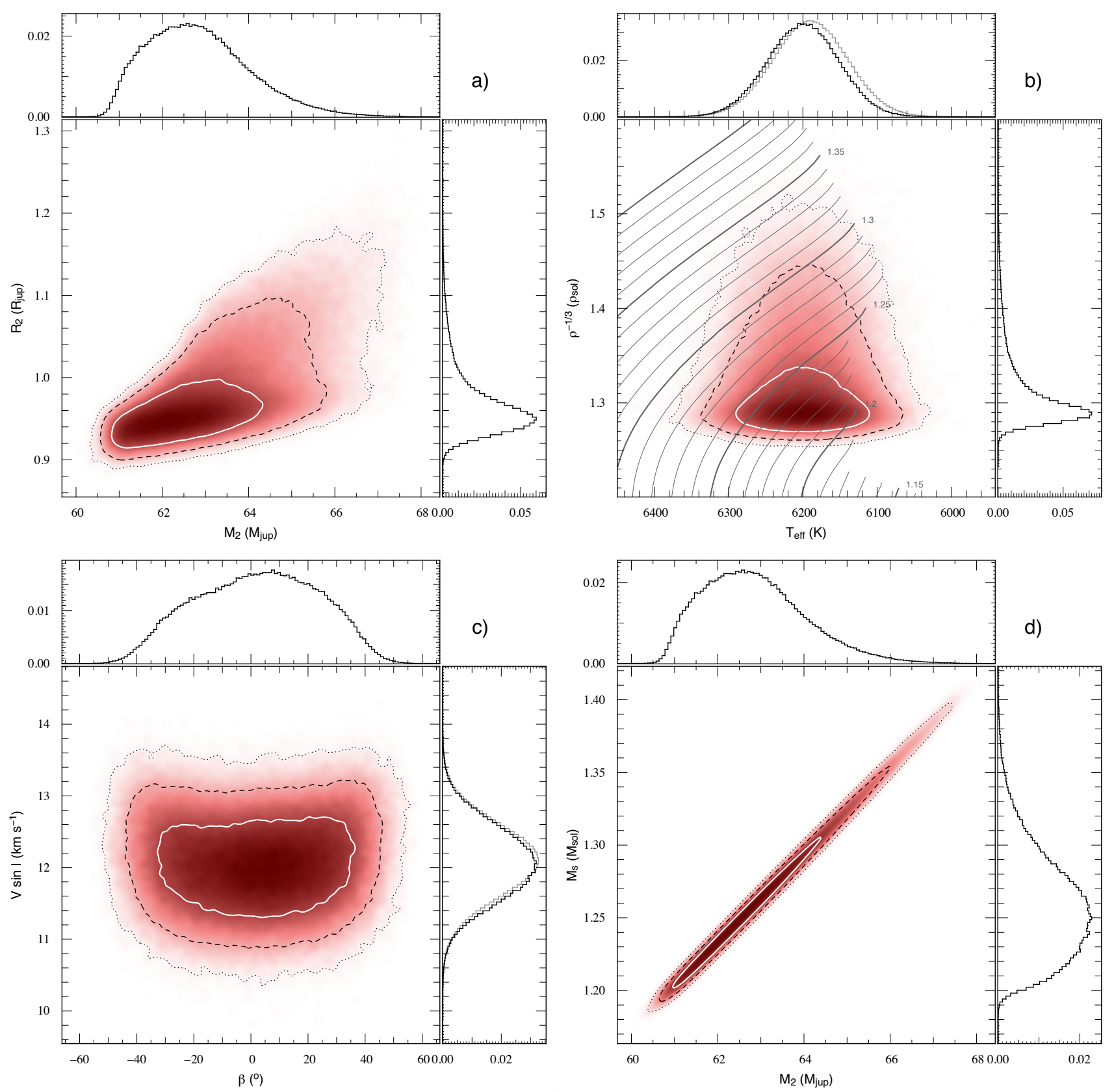

Fig. 4. WASP-30: the central panels show posterior probability-density distributions from the MCMC output, with contours at the 1-, 2- and $3-\sigma$ confidence regions. The side panels show marginalised distributions as histograms in black. Where used, the priors are shown in grey. Panel a) radius and mass of WASP-30b. b) modified Hertzsprung-Russell diagram over-plotted with the Geneva evolution tracks. Masses are indicated in $M_{\odot}$. c) $V \sin i_{\star}$ versus $\beta$ from fitting the Rossiter-McLaughlin effect. d) dependence of the secondary's mass on our incomplete knowledge of the primary mass.

acceleration is detected either. We calculated an upper constraint of $|\dot{\gamma}|<53 \mathrm{~m} \mathrm{~s}^{-1} \mathrm{yr}^{-1}$.

The second feature of interest is the Rossiter-McLaughlin effect. Because of a low impact parameter the known degeneracy between $V \sin i_{\star}, \beta$ and $b$ does not yield an unique solution (see for example Albrecht et al. 2011b; or Triaud et al. 2011). The application of a prior on $V \sin i_{\star}$, using the value measured from spectral line broadening, prevents the MCMC from searching unphysical values of $V \sin i_{\star}$. It also restricts the impact parameter $b$ from wandering too much, which otherwise was slightly affecting $\rho_{\star}$ and thus the primary's mass and radius. We thus select the solutions using that prior. Full results are available in Table 3.
WASP-30b is in a prograde orbit. Assuming an equator-on view $\left(i_{\star}=i\right)$ and using the posterior distribution obtained for the stellar radius and the $V \sin i_{\star}$, the rotation period of the star is estimated at $5.9 \pm 0.3$ days, away from synchronisation. Any attempt to use higher values for $V \sin i_{\star}$ would result in forcing the fit towards an inclined orbit.

\subsection{J1219-39b}

The overall fit for J1219-39 is good: we obtain $\chi^{2}=82.4 \pm 12.8$ for 61 radial velocity points giving $\chi_{\text {reduced }}^{2}=1.53 \pm 0.23$ without adding a jitter to the radial velocities. Errors on the photometry were adjusted to obtain a $\chi_{\text {reduced }}^{2}=1$. The imposition of priors using the Torres relation on $M_{\star}$ and $R_{\star}$ does not 
A. H. M. J. Triaud et al.: EBLM project. I.
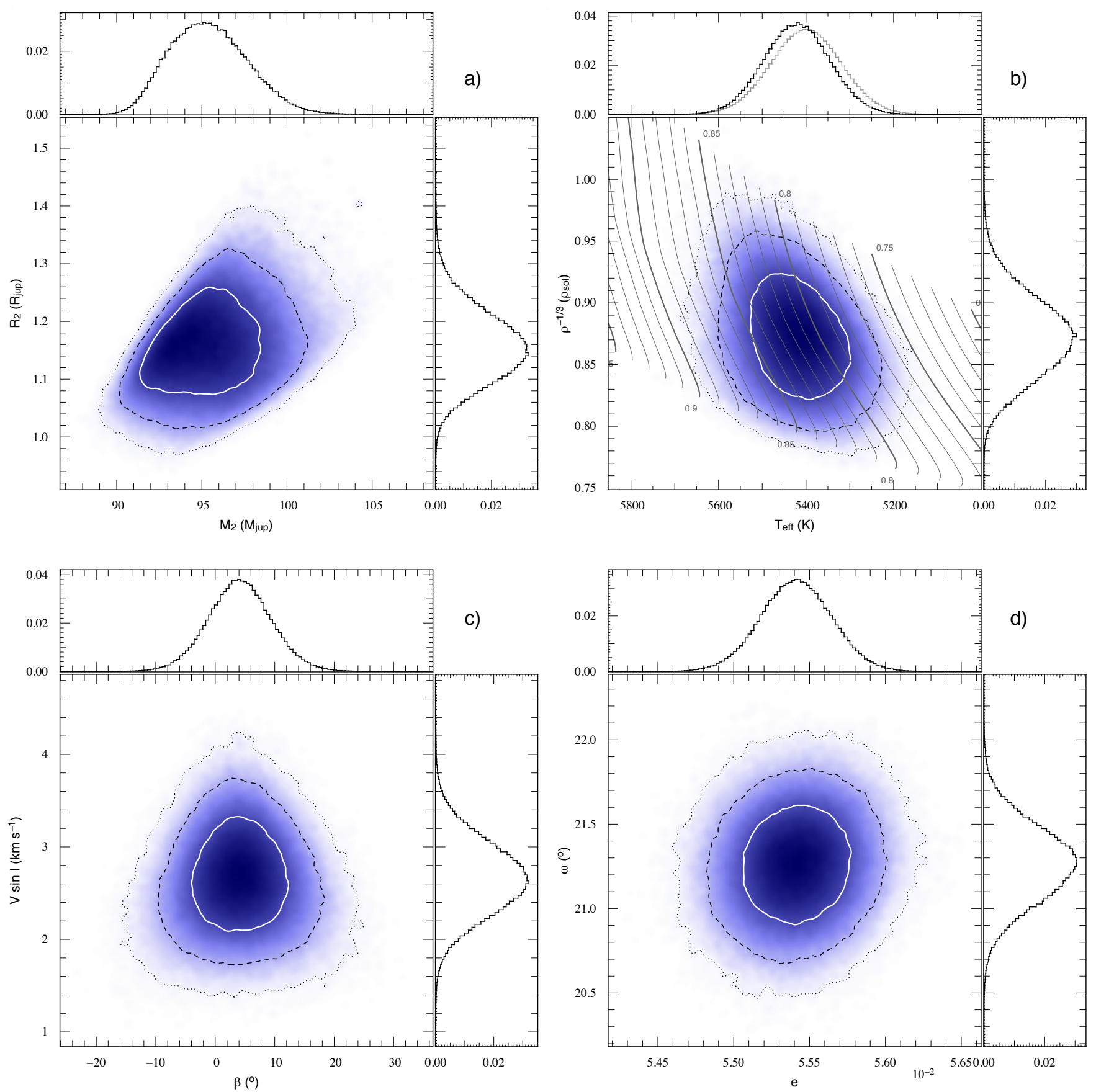

Fig. 5. J1219-39: the central panels show posterior probability-density distributions from the MCMC output, with contours at the 1-, 2- and 3- $\sigma$ confidence regions. The side panels show marginalised distributions as histograms in black. Where used, the priors are shown in grey. Panel a) radius and mass of $\mathrm{J} 1219-39 \mathrm{~b}$. b) modified Hertzsprung-Russell diagram over-plotted with the Geneva evolution tracks. Masses are indicated in $M_{\odot}$. c) $V \sin i_{\star}$ versus $\beta$ from fitting the Rossiter-McLaughlin effect. d) the eccentricity $e$ and argument of periastron $\omega$.

affect the results. We thus adopt a prior-free chain. From the eclipse and spectroscopy, without any assumptions, we obtained $\log g_{2}=5.25 \pm 0.05$ indicating that an unresolved dense object is orbiting the primary. After careful analysis we find that J1219$39 \mathrm{~A}$ is a $0.83 \pm 0.03 M_{\odot}, 0.81 \pm 0.03 R_{\odot}$ star and its companion is a low mass star, of $0.091 \pm 0.002 M_{\odot}$ and $0.117 \pm 0.006 R_{\odot}$ $\left(95 \pm 2 M_{\text {Jup }}\right.$ and $\left.1.14 \pm 0.05 R_{\text {Jup }}\right)$. The orbit is slightly eccentric ( $e=0.0554 \pm 0.0002)$ while $\beta=4^{\circ} \pm 5$, showing good spinorbit alignment. Full results are presented in Table 3. Here too we do not detect any additional acceleration and can place an upper constraint with $|\dot{\gamma}|<10 \mathrm{~m} \mathrm{~s}^{-1} \mathrm{yr}^{-1}$.
Claims of low eccentricities have been disputed in the past (Lucy \& Sweeney 1971). Now, thanks to the high precision achieved with radial velocities, it is possible to measure extremely small orbital eccentricities. As a rule of thumb, one cannot conclusively detect eccentricity if the difference between the circular and eccentric model is smaller than the RMS of the residuals. This difference can be approximated by $2 e K$. In our case we are well above that value. We nevertheless forced a circular model and find a much poorer fit reflected in a $\chi^{2}=$ $46217 \pm 304$ instead of $\chi^{2}=62.2 \pm 11.1$ for the eccentric model (on the 42 points not affected by the RM effect). 
From fitting the Rossiter-McLaughlin effect we obtain an independent (prior-free) distribution for stellar rotation peaking at $V \sin i_{\star}=2.6 \pm 0.4 \mathrm{~km} \mathrm{~s}^{-1}$. This value is significantly different from the value of $v \sin i_{\star}$ presented in Table 1 and obtained from stellar line broadening. If we use $v \sin i_{\star}$ as a prior on $V \sin i_{\star}$, the fit of the Rossiter-McLaughlin effect worsens slightly, but stays within the natural noise variability. Under this prior, the most likely value becomes $V \sin i_{\star}=3.6 \pm 0.3$, in between the independent values. This matches the value of $v \sin i_{\star}$ obtained when using a macroturbulence value from Valenti \& Fischer (2005) instead of from Bruntt et al. (2010a). We adopt the value of $V \sin i_{\star}$ found from the Rossiter-McLaughlin effect alone, as it is a directly measured value, one that can be tested against macroturbulence laws.

Assuming coplanarity $\left(i=i_{\star}\right)$ as indicated by $\beta$ and using the MCMC's posterior probabilities and the RM's $V \sin i_{\star}$, J1219-39A would have a rotation period of $15.2 \pm 2.1$ days. The solution using a prior on $V \sin i_{\star}$ gives a period of rotation of $11.7 \pm 1.0$ days, while when using instead the value of $v \sin i_{\star}$ in Table 1 we obtain $9.0 \pm 0.9$ days. Since none of these is compatible with the orbital period, we have neither synchronisation between the secondary's orbital motion and the primary's rotation, nor pseudo-synchronisation.

An analysis of the out-of-transit WASP data shows a recurring frequency at about 10.6 days on three seasons of data, presumably due to the rotation of stellar spots on the surface of the primary (see Sect. 3.1). This fourth possible rotation period is a direct observable. The discrepancy with the value obtained using the $V \sin i_{\star}$ is not understood, yet is interesting to note. Since the Rossiter-McLaughlin effect only measures the projection of the spin-orbit angle onto the sky, this could lead to presume $i_{\star}=42^{\circ} \pm 8$, indicating a spin-orbit misalignment despite $\beta$ being compatible with $0^{\circ}$ and having a good precision. The discrepancy between the different values of equatorial velocities prevents us from making a claim and warrant further comparison between the $V \sin i_{\star}$ from the Rossiter-McLaughlin and photometric rotation periods.

\section{Discussion and conclusions}

We announce the discovery of a new low-mass star whose mass and radius have been precisely measured and found to be at the junction between the stellar and substellar regimes. In addition, using observations with the CORALIE spectrograph, on the $1.2 \mathrm{~m}$ Euler Telescope, and HARPS, on the ESO $3.6 \mathrm{~m}$, we have demonstrated the detection of the Rossiter-McLaughlin effect on two objects more massive than planets. These measurements are amongst the first to be realised on such objects. They will help study the dynamical events that could have led to the formation of binary systems where both components have a large mass difference, and may also provide a useful comparison sample to the spin-orbit angle distribution of hot Jupiters, as well as helping theoretical developments in the treatment of tides, the main mechanism behind synchronisation, circularisation and realignment.

Hébrard et al. (2011) and Moutou et al. (2011) note that while hot Jupiters are usually found with a large variety of orbital angles, objects above $5 M_{\text {jup }}$ are not found on retrograde orbits. We extend the distribution of spin-orbit angle versus mass to beyond the planetary range. Albrecht et al. (2012) instead of the mass plot the mass ratio against the projected spin-orbit angle, which makes more physical sense. Both of our objects have projected spin-orbit angles aligned with their primaries', further confirming that trend. Being around stars colder than $6250 \mathrm{~K}$, they also reinforce the pattern shown by Winn et al. (2010) between orbital inclination and the primary's effective temperature. The age of WASP-30A and the alignment of WASP-30b also helps strengthen the pattern claimed in Triaud (2011b) that systems older than 2.5-3 Gyr appear predominantly aligned. It is interesting to note that while J1219-39b is on a slightly eccentric orbit, its projected orbital spin is aligned with its primary's rotational spin. This gives observational evidence that orbital realignment may be faster than orbital circularisation for these objects, in opposition with planets. Final tidal equilibrium has not been reached for either system as WASP-30A is not synchronised and J1219-39b is not circularised (nor synchronised) (Hut 1980). It is to be noted that those conclusions use the projected spin-orbit angle, and not the real orbital obliquity $\psi$. In the case of J1219-39b, the discrepancy between the photometric period and the one inferred from the $V \sin i_{\star}$, could be indicative of a spin-orbit misalignment that, when confirmed, would bring support to a formation scenario as outlined in Mazeh \& Shaham (1979).

While not being the primary objective of this paper, we present a method to analyse, in a global manner, eclipse photometry, the radial-velocity reflex motion, and the RossiterMcLaughlin effect, for objects more massive than planets, in order to obtain precise estimates of the mass, radius and orbital parameters of SB1s. The precision of a few percent that we obtain comes from our use of $\rho_{\star}$, the mean stellar density, instead of the more traditional $\log g$ when interpolating inside the stellar evolution tracks. This interpolation gives us precise values for the primary's stellar parameters which are used to estimate the secondary's parameters. We can check our method by deducing $\log g_{\star}$ and comparing them to their spectral counterpart. The values are in very good agreement and fall within the errors of the spectral method (see Table 6). This method also allows us to estimate ages from reading the stellar tracks, something important in the case of close binaries where gyrochronology cannot be trusted owing to the tidal evolution of the system.

Our low-mass eclipsing objects have very similar surface gravities, but, located on opposite sides of the brown-dwarf limit, have sizes dominated by different physics (Baraffe et al. 2003). Plotting the posterior probability distribution for the mass and radius on dust-free theoretical mass-radius relations (Baraffe et al. 2003, 1998) in Fig. 7, we observe that WASP-30b is between the 0.5 and 1 Gyr tracks, suggesting the object is fairly young (and thus luminous, which should cause a measurable secondary eclipse). This is at odds with the age of the primary, which we have found to be older than 2 Gyr with $99 \%$ confidence, with a best age of $3.4 \pm 0.4$ Gyr (Fig. 6).

We could explain this radius anomaly if the object has been inflated in the same manner that has been observed for hot Jupiters thanks to the high irradiation received from its primary (Demory \& Seager 2011). The exact physical causes are still being debated. It may also be that energy has been stored inside the object if it circularised from a previously highly eccentric orbit (Mazeh \& Shaham 1979; Fabrycky \& Tremaine 2007; Heller et al. 2010). Alternatively, one could use models involving different Helium contents, or including the formation of dust clouds. Those alter the evolution track significantly in that part of the diagram and can be made to fit the mass, radius, age and metallicity of our object (Burrows et al. 2011). WASP-30b's mass is interesting in that it is close to the minimum of the tracks presented in Baraffe et al. (2003) and displayed in Fig. 7. The massradius posterior distribution of $\mathrm{J} 1219-39 \mathrm{~b}$ is compatible with the 10-Gyr theoretical line. Better photometry would help to reduce the confidence region. This analysis will be the subject of 


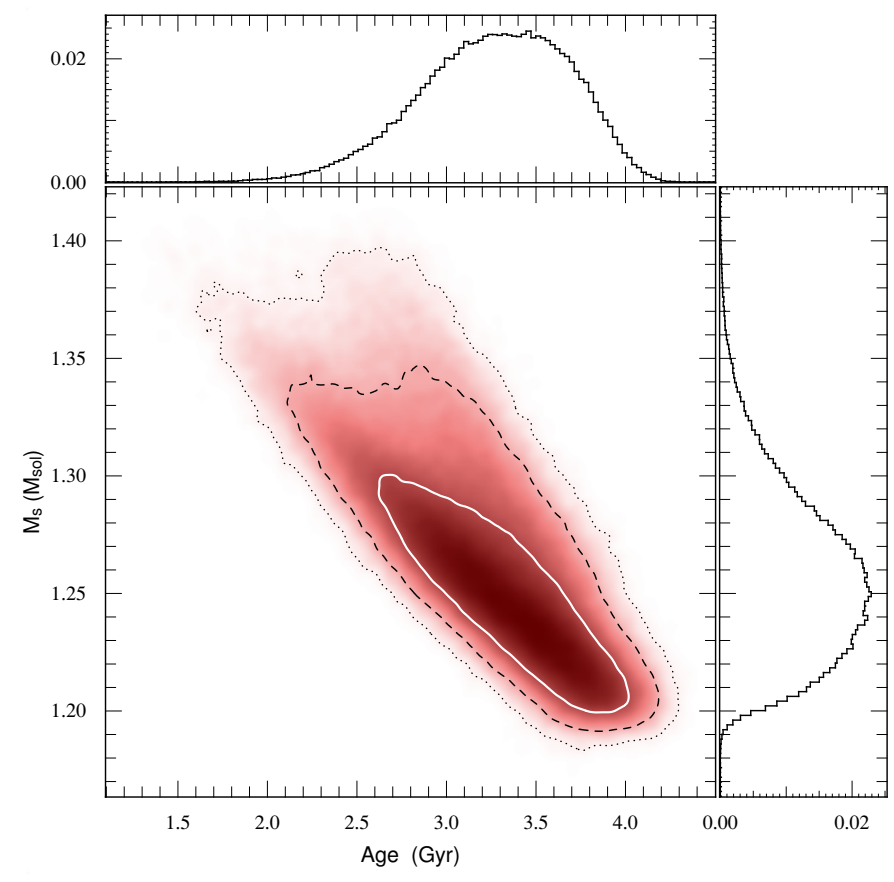

Fig. 6. The central panel shows the posterior probability-density distribution for stellar age against stellar mass for WASP-30. The contours show the 1-, 2- and 3- $\sigma$ confidence regions; the side panels show the marginalised distributions as histograms. The data derive from interpolating using the mean stellar density, $T_{\mathrm{eff}}$ and metallicity, into the Geneva stellar evolution tracks (Mowlavi et al. 2012).

subsequent papers (Hebb et al., in prep.). One could object that our analysis does not take into account the fact that both WASP$30 \mathrm{~b}$ and J1219-39b are self luminous. Even in the case that they had the same effective temperature as their primaries, the overall contamination cannot be larger than their relative sizes $\sim 1 \%$, lower than our current precision.

We would like to attract the attention on the fact that both those objects have sizes entirely compatible with those of hot Jupiters. While hot Jupiters are often inflated, Jupiter-mass planets at longer periods no longer are (Demory \& Seager 2011). It would then be expected that many of the planet candidates published by the space mission Kepler with inflated radii ( $\left.>1.2 R_{\mathrm{Jup}}\right)$ and periods longer than 10-15 days could be objects similar to WASP-30b and J1219-39b. While not being planets, they are of great interest for their masses, radii and orbital parameters.

Finally, the Rossiter-McLaughlin effect has recently been used almost exclusively to measure planetary orbital planes. Observing it for binary stars extends that work by bridging the gap in mass ratio between planetary and stellar systems. Comparison between low-mass binaries in our case with highermass binaries as in the BANANA survey (Albrecht et al. 2011a) will permit us to test different regimes of binary formation and tidal interactions.

Yet more information still lies in the study of this RV anomaly. Its use in the beginning of the 20th century was primarily to measure the rotation of stars. As seen in this paper, there is a discrepancy between the $v \sin i_{\star}$ values obtained by using calibration of the macroturbulence and the directly measured $V \sin i_{\star}$ from the Rossiter-McLaughlin effect. This was also pointed out in Triaud et al. (2011) in the case of WASP-23, and by Brown et al. (2012) in the case of WASP-16. Both those systems, like J1291-39, contain K dwarfs, and both had their spectroscopic $v \sin i_{\star}$ overestimated compared to the value obtained

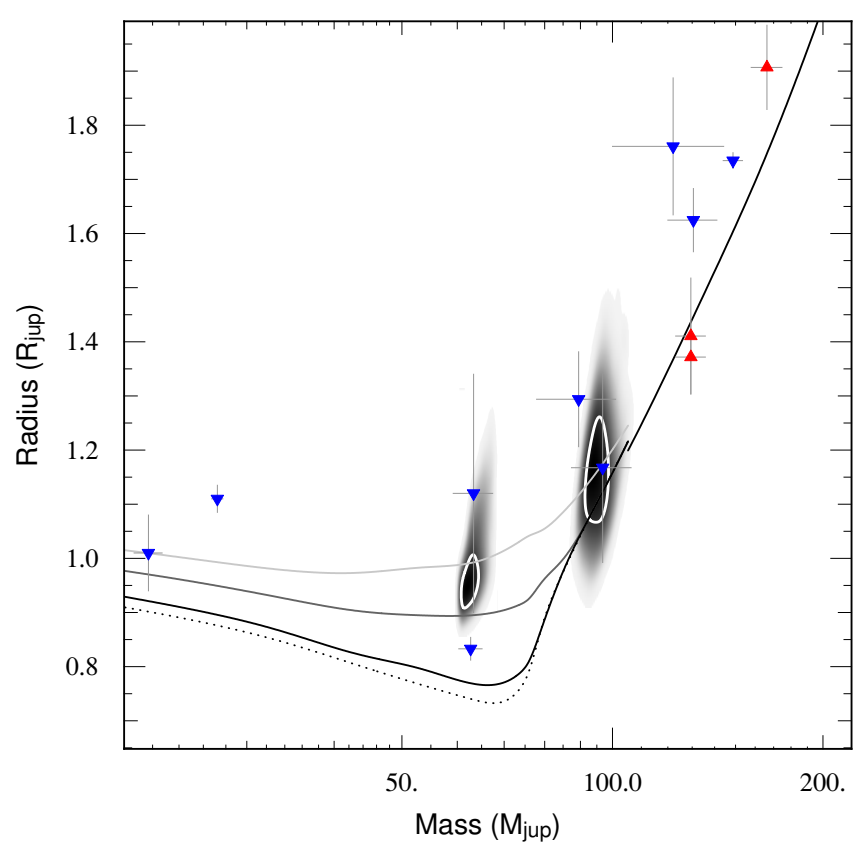

Fig. 7. Mass-radius diagram for heavy planets, brown dwarfs and low-mass stars. The radius axis corresponds to the size range of Jupitermass planets discovered so far. Inverted blue triangles show eclipsing/transiting SB1s, upright red triangles denote interferometric measurements. The two $\left(M_{2}, R_{2}\right)$ posterior probability density distributions for WASP-30b and J1219-39b are drawn in grey with their $1-\sigma$ confidence regions in white. Models by Baraffe et al. $(2003,1998)$ are also displayed with ages $5 \mathrm{Gyr}$ (black), $1 \mathrm{Gyr}$ (dark grey), $500 \mathrm{Myr}$ (light grey) and $10 \mathrm{Gyr}$ (dotted). Models are for $[\mathrm{M} / \mathrm{H}]=0$. Observational data were taken from Lane et al. (2001); Ségransan et al. (2003); Pont et al. (2005a,b, 2006); Beatty et al. (2007); Deleuil et al. (2008); Demory et al. (2009); Bouchy et al. (2011); Johnson et al. (2011); Ofir et al. (2012); Siverd et al. (2012).

via the Rossiter-McLaughlin effect. Since we compute the spinorbit angle $\beta$ and the $V \sin i_{\star}$, we have strong constraints on the coplanarity of the system and rotation velocity of the primary; thanks to the transit/eclipse geometry, we obtain accurate and precise masses and radii for both the primary and the secondary. Combining all this information and collecting many measurements, we will be able to test which of the macroturbulence laws one should use. Should none apply, inserting the observed $V \sin i_{\star}$ values as input parameters in spectral line analyses we will have the capacity to measure macroturbulence directly. Observing the Rossiter-McLaughlin effect is thus not just about glimpsing into the past dynamical history of systems, but can also become an important tool for understanding stellar physics better.

Nota Bene We used the UTC time standard and Barycentric Julian dates in our analysis. Our results are based on the equatorial solar and Jovian radii and masses taken from Allen's astrophysical quantities.

Acknowledgements. The authors would like to acknowledge the use of ADS and of Simbad at CDS. We also would like to attract attention on the help and kind attention of the ESO staff at La Silla and at the guesthouse in Santiago as well as on the dedication of the many observers whose efforts during many nights were required to obtain all the data presented here. Special thanks go to our programmers and their wonderful automatic Data Reduction Software, allowing us to observe live (!) an object transiting its primary (be it planet, brown dwarf or M dwarf), thus makes observing so much more exciting. We thank 
Kris Hełminiak for commenting on the draft and generally for being a nice guy. Thanks also go to Brice-Oliver Demory for his help with regards with interferometric masses and radii measurements of low-mass stars and the inspiration he provided to AT for starting to seek brown dwarfs, which led to the discovery of those many low-mass binaries. This work is supported financially by the Swiss Fond National de Recherche Scientifique. We wish to thank the referee for reviewing the science and for his help in making this paper clearer.

\section{References}

Albrecht, S., Reffert, S., Snellen, I., Quirrenbach, A., \& Mitchell, D. S. 2007, A\&A, 474, 565

Albrecht, S., Reffert, S., Snellen, I. A. G., \& Winn, J. N. 2009, Nature, 461, 373

Albrecht, S., Winn, J. N., Carter, J. A., Snellen, I. A. G., \& de Mooij, E. J. W. 2011a, ApJ, 726, 68

Albrecht, S., Winn, J. N., Johnson, J. A., et al. 2011b, ApJ, 738, 50

Albrecht, S., Winn, J. N., Johnson, J. A., et al. 2012, ApJ, 757, 18

Anderson, D. R., Collier Cameron, A., Hellier, C., et al. 2011a, A\&A, 531, A60

Anderson, D. R., Collier Cameron, A., Hellier, C., et al. 2011b, ApJ, 726, L19

Baraffe, I., Chabrier, G., Allard, F., \& Hauschildt, P. H. 1998, A\&A, 337, 403

Baraffe, I., Chabrier, G., Barman, T. S., Allard, F., \& Hauschildt, P. H. 2003, A\&A, 402, 701

Baranne, A., Queloz, D., Mayor, M., et al. 1996, A\&AS, 119, 373

Beatty, T. G., Fernández, J. M., Latham, D. W., et al. 2007, ApJ, 663, 573

Bentley, S. J., Smalley, B., Maxted, P. F. L., et al. 2009, A\&A, 508, 391

Bolmont, E., Raymond, S. N., \& Leconte, J. 2011, A\&A, 535, A94

Bolmont, E., Raymond, S. N., Leconte, J., \& Matt, S. P. 2012, A\&A, 544, A124

Bouchy, F., Deleuil, M., Guillot, T., et al. 2011, A\&A, 525, A68

Brown, D. J. A., Cameron, A. C., Anderson, D. R., et al. 2012, MNRAS, 423, 1503

Bruntt, H., De Cat, P., \& Aerts, C. 2008, A\&A, 478, 487

Bruntt, H., Bedding, T. R., Quirion, P.-O., et al. 2010a, MNRAS, 405, 1907

Bruntt, H., Deleuil, M., Fridlund, M., et al. 2010b, A\&A, 519, A51

Burrows, A., Heng, K., \& Nampaisarn, T. 2011, ApJ, 736, 47

Caballero, J. A., Béjar, V. J. S., Rebolo, R., et al. 2007, A\&A, 470, 903

Claret, A. 2004, A\&A, 428, 1001

Collier Cameron, A., Bouchy, F., Hébrard, G., et al. 2007a, MNRAS, 375, 951

Collier Cameron, A., Wilson, D. M., West, R. G., et al. 2007b, MNRAS, 380, 1230

Deleuil, M., Deeg, H. J., Alonso, R., et al. 2008, A\&A, 491, 889

Demory, B.-O., \& Seager, S. 2011, ApJS, 197, 12

Demory, B.-O., Ségransan, D., Forveille, T., et al. 2009, A\&A, 505, 205

Desmet, M., Frémat, Y., Baudin, F., et al. 2010, MNRAS, 401, 418

Doyle, L. R., Carter, J. A., Fabrycky, D. C., et al. 2011, Science, 333, 1602

Enoch, B., Collier Cameron, A., Parley, N. R., \& Hebb, L. 2010, A\&A, 516, A33

Fabrycky, D., \& Tremaine, S. 2007, ApJ, 669, 1298

Fernandez, J. M., Latham, D. W., Torres, G., et al. 2009, ApJ, 701, 764

Ford, E. B. 2006, ApJ, 642, 505

Gaudi, B. S., \& Winn, J. N. 2007, ApJ, 655, 550

Gillon, M., Anderson, D. R., Triaud, A. H. M. J., et al. 2009a, A\&A, 501, 785

Gillon, M., Smalley, B., Hebb, L., et al. 2009b, A\&A, 496, 259

Gillon, M., Jehin, E., Magain, P., et al. 2011, Detection and Dynamics of

Transiting Exoplanets, St. Michel l'Observatoire, France, eds. F. Bouchy,

R. Díaz, \& C. Moutou, EPJ Web Conf., 11, 6002

Gillon, M., Triaud, A. H. M. J., Fortney, J. J., et al. 2012, A\&A, 542, A4

Giménez, A. 2006, ApJ, 650, 408

Gray, D. F. 2008, The Observation and Analysis of Stellar Photospheres, ed. D. F. Gray

Hebb, L., Collier-Cameron, A., Loeillet, B., et al. 2009, ApJ, 693, 1920

Hébrard, G., Bouchy, F., Pont, F., et al. 2008, A\&A, 488, 763

Hébrard, G., Ehrenreich, D., Bouchy, F., et al. 2011, A\&A, 527, L11

Heller, R., Jackson, B., Barnes, R., Greenberg, R., \& Homeier, D. 2010, A\&A, 514, A 22

Hilditch, R. W. 2001, An Introduction to Close Binary Stars, ed. R. W. Hilditch Holt, J. R. 1893, Astro-Physics, XII, 646

Hosokawa, Y. 1953, PASJ, 5, 88

Hube, D. P., \& Couch, J. S. 1982, Ap\&SS, 81, 357

Hut, P. 1980, A\&A, 92, 167

Hut, P. 1981, A\&A, 99, 126

Jehin, E., Gillon, M., Queloz, D., et al. 2011, The Messenger, 145, 2

Johnson, J. A., Apps, K., Gazak, J. Z., et al. 2011, ApJ, 730, 79

Kopal, Z. 1942a, ApJ, 96, 399

Kopal, Z. 1942b, Proc. Nat. Acad. Sci., 28, 133
Kozai, Y. 1962, AJ, 67, 579

Lane, B. F., Boden, A. F., \& Kulkarni, S. R. 2001, ApJ, 551, L81

López-Morales, M. 2007, ApJ, 660, 732

Lovis, C., \& Pepe, F. 2007, A\&A, 468, 1115

Lucy, L. B., \& Sweeney, M. A. 1971, AJ, 76, 544

Magain, P. 1984, A\&A, 134, 189

Malmberg, D., Davies, M. B., \& Heggie, D. C. 2011, MNRAS, 411, 859

Malmberg, D., de Angeli, F., Davies, M. B., et al. 2007, MNRAS, 378, 1207

Mandel, K., \& Agol, E. 2002, ApJ, 580, L171

Matsumura, S., Peale, S. J., \& Rasio, F. A. 2010a, ApJ, 725, 1995

Matsumura, S., Thommes, E. W., Chatterjee, S., \& Rasio, F. A. 2010b, ApJ, 714, 194

Maxted, P. F. L., Anderson, D. R., Collier Cameron, A., et al. 2011, PASP, 123, 547

Mayor, M., Udry, S., Lovis, C., et al. 2009, A\&A, 493, 639

Mazeh, T., \& Shaham, J. 1979, A\&A, 77, 145

McLaughlin, D. B. 1924, ApJ, 60, 22

Morales, J. C., Gallardo, J., Ribas, I., et al. 2010, ApJ, 718, 502

Morales, J. C., Ribas, I., \& Jordi, C. 2008, A\&A, 478, 507

Morales, J. C., Ribas, I., Jordi, C., et al. 2009, ApJ, 691, 1400

Mordasini, C., Alibert, Y., \& Benz, W. 2009, A\&A, 501, 1139

Moutou, C., Díaz, R. F., Udry, S., et al. 2011, A\&A, 533, A113

Mowlavi, N., Eggenberger, P., Meynet, G., et al. 2012, A\&A, 541, A41

Munari, U., \& Zwitter, T. 1997, A\&A, 318, 269

Nagasawa, M., Ida, S., \& Bessho, T. 2008, ApJ, 678, 498

Naoz, S., Farr, W. M., Lithwick, Y., Rasio, F. A., \& Teyssandier, J. 2011, Nature, 473, 187

Ofir, A., Gandolfi, D., Buchhave, L., et al. 2012, MNRAS, 423, L1

Pepe, F., Mayor, M., Rupprecht, G., et al. 2002, The Messenger, 110, 9

Pollacco, D. L., Skillen, I., Collier Cameron, A., et al. 2006, PASP, 118, 1407

Pont, F., Bouchy, F., Melo, C., et al. 2005a, A\&A, 438, 1123

Pont, F., Melo, C. H. F., Bouchy, F., et al. 2005b, A\&A, 433, L21

Pont, F., Moutou, C., Bouchy, F., et al. 2006, A\&A, 447, 1035

Queloz, D., Eggenberger, A., Mayor, M., et al. 2000, A\&A, 359, L13

Rasio, F. A., \& Ford, E. B. 1996, Science, 274, 954

Rossiter, R. A. 1924, ApJ, 60, 15

Schlesinger, F. 1910, Publications of the Allegheny Observatory of the University of Pittsburgh, 1, 123

Ségransan, D., Kervella, P., Forveille, T., \& Queloz, D. 2003, A\&A, 397, L5

Sestito, P., \& Randich, S. 2005, A\&A, 442, 615

Siverd, R. J., Beatty, T. G., Pepper, J., et al. 2012, ApJ, submitted [arXiv: 1206.1635]

Slettebak, A. 1985, in Calibration of Fundamental Stellar Quantities, eds. D. S. Hayes, L. E. Pasinetti, \& A. G. D. Philip, IAU Symp., 111, 163

Southworth, J., Zucker, S., Maxted, P. F. L., \& Smalley, B. 2004, MNRAS, 355, 986

Sozzetti, A., Torres, G., Charbonneau, D., et al. 2007, ApJ, 664, 1190

Stetson, P. B. 1987, PASP, 99, 191

Struve, O. 1950, Stellar evolution, an exploration from the observatory, ed. O. Struve

Thies, I., Kroupa, P., Goodwin, S. P., Stamatellos, D., \& Whitworth, A. P. 2011, MNRAS, 417, 1817

Tokovinin, A., Thomas, S., Sterzik, M., \& Udry, S. 2006, A\&A, 450, 681

Torres, G., Andersen, J., \& Giménez, A. 2010, A\&ARv, 18, 67

Triaud, A. H. M. J. 2011a, Ph.D. Thesis, Observatoire Astronomique de l'Universite de Geneve,

http://archive-ouverte.unige.ch/unige: 18065

Triaud, A. H. M. J. 2011b, A\&A, 534, L6

Triaud, A. H. M. J., Queloz, D., Bouchy, F., et al. 2009, A\&A, 506, 377

Triaud, A. H. M. J., Collier Cameron, A., Queloz, D., et al. 2010, A\&A, 524, A25

Triaud, A. H. M. J., Queloz, D., Hellier, C., et al. 2011, A\&A, 531, A24

Udalski, A. 2007, in Transiting Extrapolar Planets Workshop, ed. C. Afonso,

D. Weldrake, \& T. Henning, ASP Conf. Ser., 366, 51

Valenti, J. A., \& Fischer, D. A. 2005, ApJS, 159, 141

Winn, J. N., Noyes, R. W., Holman, M. J., et al. 2005, ApJ, 631, 1215

Winn, J. N., Johnson, J. A., Albrecht, S., et al. 2009, ApJ, 703, L99

Winn, J. N., Fabrycky, D., Albrecht, S., \& Johnson, J. A. 2010, ApJ, 718, L145

Winn, J. N., Albrecht, S., Johnson, J. A., et al. 2011, ApJ, 741, L1

Worek, T. F. 1996, PASP, 108, 962

Wu, Y., \& Lithwick, Y. 2011, ApJ, 735, 109

Wu, Y., Murray, N. W., \& Ramsahai, J. M. 2007, ApJ, 670, 820

Young, P., \& Schneider, D. P. 1980, ApJ, 238, 955

Zahn, J.-P. 1977, A\&A, 57, 383

Pages 13 to 16 are available in the electronic edition of the journal at http://www . aanda.org 
A. H. M. J. Triaud et al.: EBLM project. I.

\section{Appendix A: Journal of observations}

Table A.1. CORALIE radial velocities on WASP-30.

\begin{tabular}{|c|c|c|c|c|c|}
\hline & $\begin{array}{c}\text { JDB-2 } 400000 \\
\text { day }\end{array}$ & $\begin{array}{c}\mathrm{RV} \\
\mathrm{m} \mathrm{s}^{-1}\end{array}$ & $\begin{array}{c}1 \sigma \text { error } \\
\mathrm{m} \mathrm{s}^{-1}\end{array}$ & $\begin{array}{c}\text { Bissector span } \\
\mathrm{m} \mathrm{s}^{-1}\end{array}$ & $\begin{array}{c}\text { Exposition time } \\
\mathrm{s}\end{array}$ \\
\hline & 55009.906531 & 14.26750 & 0.03161 & 0.08307 & 1800.687 \\
\hline & 55040.872183 & 1.29532 & 0.04933 & -0.18938 & 1800.683 \\
\hline & 55092.697730 & 14.34312 & 0.04450 & 0.04157 & 1800.680 \\
\hline & 55095.689403 & 5.16045 & 0.02890 & -0.04933 & 1800.678 \\
\hline & 55096.547613 & 12.97375 & 0.03861 & -0.01846 & 1800.680 \\
\hline & 55096.871175 & 14.44650 & 0.04125 & 0.00031 & 1800.680 \\
\hline & 55097.535138 & 12.50573 & 0.04066 & 0.04896 & 1800.680 \\
\hline & 55097.873525 & 9.72874 & 0.04880 & 0.04062 & 1800.680 \\
\hline & 55098.553830 & 3.34309 & 0.03992 & -0.00745 & 1800.678 \\
\hline & 55113.520897 & 14.44839 & 0.03112 & -0.13446 & 2700.520 \\
\hline & 55113.587712 & 14.55705 & 0.03513 & -0.01233 & 1800.676 \\
\hline & 55113.611079 & 14.50745 & 0.03642 & -0.01244 & 1800.676 \\
\hline & 55113.634342 & 14.57909 & 0.03446 & -0.10343 & 1800.676 \\
\hline & 55113.657605 & 14.48377 & 0.03133 & -0.03359 & 1800.676 \\
\hline & 55113.680879 & 14.52860 & 0.02992 & -0.06087 & 1800.677 \\
\hline & 55113.704143 & 14.50256 & 0.03056 & -0.06218 & 1800.677 \\
\hline & 55372.894609 & 3.43134 & 0.02395 & 0.02263 & 1800.692 \\
\hline & 55374.882333 & 11.71436 & 0.02861 & -0.07726 & 1800.692 \\
\hline & 55375.912731 & 13.35947 & 0.02948 & -0.00708 & 1800.690 \\
\hline & 55376.943164 & 4.24058 & 0.02456 & 0.02991 & 1800.690 \\
\hline \# & 55406.669984 & 21.10817 & 0.15149 & -0.69141 & 600.570 \\
\hline \# & 55406.679382 & 21.19725 & 0.16601 & -0.56823 & 600.570 \\
\hline \# & 55406.688770 & 21.08917 & 0.17616 & 0.44692 & 600.570 \\
\hline \# & 55406.698180 & 20.98105 & 0.16437 & -0.31414 & 600.570 \\
\hline \# & 55406.707903 & 21.09235 & 0.16569 & -0.88959 & 600.570 \\
\hline \# & 55406.717302 & 21.04424 & 0.14780 & 0.31012 & 600.570 \\
\hline \# & 55406.726712 & 21.04202 & 0.19272 & -1.53008 & 600.570 \\
\hline \# & 55406.736354 & 3.44740 & 0.16206 & 4.13793 & 600.570 \\
\hline \# & 55406.745753 & 3.99157 & 0.17244 & 2.36916 & 600.570 \\
\hline \# & 55406.755152 & 21.61630 & 0.16635 & 0.06005 & 600.570 \\
\hline \# & 55406.767595 & 1.62258 & 0.11359 & -0.28920 & 600.570 \\
\hline \# & 55406.776994 & 4.15874 & 0.16749 & 5.89641 & 600.570 \\
\hline \multirow[t]{15}{*}{ \# } & 55406.786520 & 21.54117 & 0.16369 & -0.26250 & 600.570 \\
\hline & 55483.592040 & 14.52330 & 0.02797 & 0.05140 & 1800.686 \\
\hline & 55484.555214 & 8.71115 & 0.03915 & 0.01610 & 1200.133 \\
\hline & 55484.571637 & 8.57906 & 0.03658 & -0.04975 & 1200.133 \\
\hline & 55484.591612 & 8.35231 & 0.02803 & -0.13637 & 1800.685 \\
\hline & 55484.614887 & 8.11803 & 0.02732 & -0.11433 & 1800.685 \\
\hline & 55484.638161 & 7.80482 & 0.02456 & 0.05655 & 1800.685 \\
\hline & 55484.661528 & 7.56473 & 0.02306 & 0.08690 & 1800.686 \\
\hline & 55484.684814 & 7.34139 & 0.02417 & -0.07061 & 1800.686 \\
\hline & 55484.708112 & 7.13547 & 0.02518 & -0.04579 & 1800.686 \\
\hline & 55484.731432 & 6.95050 & 0.02617 & -0.02408 & 1800.686 \\
\hline & 55484.757021 & 6.68697 & 0.02934 & 0.03535 & 1800.686 \\
\hline & 55485.577214 & 1.43623 & 0.04333 & 0.12378 & 900.856 \\
\hline & 55485.772876 & 1.44188 & 0.04730 & 0.05507 & 900.856 \\
\hline & 55486.755271 & 8.38843 & 0.04952 & 0.00574 & 900.856 \\
\hline
\end{tabular}

Notes. Points excluded from the analysis are marked by \#. 
Table A.2. HARPS radial velocities on WASP-30.

\begin{tabular}{lcccc}
\hline \hline JDB-2 400 000 & $\begin{array}{c}\text { RV } \\
\mathrm{m} \mathrm{s}^{-1}\end{array}$ & $\begin{array}{c}1 \sigma \text { error } \\
\mathrm{m} \mathrm{s}^{-1}\end{array}$ & $\begin{array}{c}\text { Bissector span } \\
\mathrm{m} \mathrm{s}^{-1}\end{array}$ & $\begin{array}{c}\text { Exposition time } \\
\mathrm{s}\end{array}$ \\
\hline 55458.801056 & 14.31817 & 0.01359 & -0.02473 & 900.001 \\
55458.863070 & 14.18789 & 0.01721 & -0.02845 & 900.000 \\
55459.584455 & 8.92639 & 0.01303 & -0.04849 & 900.000 \\
55459.596585 & 8.79993 & 0.01243 & -0.04828 & 900.001 \\
55459.608645 & 8.71706 & 0.01227 & 0.01562 & 900.001 \\
55459.619941 & 8.59953 & 0.01115 & -0.03980 & 900.001 \\
55459.630832 & 8.51616 & 0.01139 & -0.02751 & 900.001 \\
55459.641619 & 8.39399 & 0.01172 & 0.03252 & 900.001 \\
55459.652406 & 8.32565 & 0.01163 & -0.09640 & 900.001 \\
55459.663193 & 8.19032 & 0.01169 & -0.08725 & 900.001 \\
55459.674084 & 8.08172 & 0.01227 & -0.07852 & 900.001 \\
55459.684663 & 7.92218 & 0.01182 & -0.00659 & 900.001 \\
55459.695554 & 7.81028 & 0.01274 & -0.01962 & 900.001 \\
55459.706341 & 7.70502 & 0.01233 & 0.02461 & 900.001 \\
55459.717140 & 7.57160 & 0.01221 & 0.07484 & 900.000 \\
55459.727822 & 7.46093 & 0.01280 & 0.06383 & 900.000 \\
55459.738714 & 7.36897 & 0.01172 & 0.00101 & 900.001 \\
55459.749709 & 7.26729 & 0.01124 & -0.06186 & 900.001 \\
55459.760183 & 7.15544 & 0.01068 & -0.00859 & 900.000 \\
55459.771086 & 7.09373 & 0.01130 & 0.02301 & 900.001 \\
55459.781769 & 6.97189 & 0.01063 & -0.01718 & 900.001 \\
55459.792938 & 6.87136 & 0.01065 & -0.00295 & 900.001 \\
55459.803516 & 6.76433 & 0.01139 & 0.03844 & 900.001 \\
55459.814199 & 6.65709 & 0.01154 & 0.00489 & 900.001 \\
55459.824986 & 6.53058 & 0.01294 & 0.06997 & 900.001 \\
55459.836190 & 6.42898 & 0.01362 & -0.01800 & 900.000 \\
55459.846352 & 6.34038 & 0.01442 & 0.06821 & 900.001 \\
55459.857555 & 6.18629 & 0.01672 & 0.11131 & 900.001 \\
55460.555502 & 0.55393 & 0.02949 & 1.38692 & 900.000 \\
55462.734059 & 14.44197 & 0.01434 & -0.08221 & 900.000 \\
55462.837403 & 14.44747 & 0.01748 & -0.08063 & 900.001 \\
55463.575646 & 10.48865 & 0.01369 & -0.14413 & 900.001 \\
55464.554164 & 2.07849 & 0.01635 & 0.08127 & 900.001 \\
55464.754091 & 1.42238 & 0.01348 & -0.01207 & 900.000 \\
55464.821243 & 1.20493 & 0.02133 & 0.20388 & 900.001 \\
55465.751553 & 6.19770 & 0.01200 & 0.00173 & 1200.001 \\
55465.836795 & 6.99038 & 0.02404 & -0.22238 & 900.001 \\
\hline & & & & \\
\hline
\end{tabular}


A. H. M. J. Triaud et al.: EBLM project. I.

Table A.3. CORALIE radial velocities on J1219-39.

\begin{tabular}{|c|c|c|c|c|c|}
\hline & $\begin{array}{c}\text { JDB-2 } 400000 \\
\text { day }\end{array}$ & $\begin{array}{c}\mathrm{RV} \\
\mathrm{m} \mathrm{s}^{-1}\end{array}$ & $\begin{array}{c}1 \sigma \text { error } \\
\mathrm{m} \mathrm{s}^{-1}\end{array}$ & $\begin{array}{l}\text { Bissector span } \\
\mathrm{m} \mathrm{s}^{-1}\end{array}$ & $\begin{array}{c}\text { Exposition time } \\
\mathrm{s}\end{array}$ \\
\hline & 54682.496675 & 23.53814 & 0.00542 & -0.02097 & 1800.678 \\
\hline & 54815.860532 & 35.05819 & 0.00643 & -0.02585 & 1500.417 \\
\hline & 54816.853869 & 26.13893 & 0.00594 & -0.03457 & 1800.677 \\
\hline & 55041.524239 & 25.66908 & 0.00662 & -0.01387 & 1800.682 \\
\hline & 55241.863326 & 33.73800 & 0.00602 & 0.00296 & 900.845 \\
\hline \multirow[t]{3}{*}{ \# } & 55299.811179 & 37.46028 & 0.02509 & 0.04919 & 1800.689 \\
\hline & 55300.613772 & 43.84828 & 0.00792 & -0.03227 & 900.857 \\
\hline & 55301.784939 & 42.61375 & 0.00748 & -0.01918 & 900.858 \\
\hline \multirow[t]{5}{*}{ \# } & 55302.558918 & 35.39085 & 0.07415 & 0.00307 & 900.859 \\
\hline & 55305.684930 & 29.22708 & 0.00654 & -0.01949 & 900.859 \\
\hline & 55308.790500 & 40.60277 & 0.00877 & -0.03277 & 600.571 \\
\hline & 55310.651007 & 24.53962 & 0.00740 & -0.01631 & 600.570 \\
\hline & 55329.678564 & 34.46613 & 0.01404 & 0.02957 & 600.570 \\
\hline \multirow[t]{5}{*}{ \# } & 55329.687962 & 34.41273 & 0.02722 & 0.00544 & 600.570 \\
\hline & 55329.720217 & 33.98013 & 0.00859 & 0.00912 & 900.857 \\
\hline & 55329.732913 & 33.86562 & 0.00933 & -0.00727 & 900.857 \\
\hline & 55329.744070 & 33.75440 & 0.01145 & 0.00512 & 600.570 \\
\hline & 55329.755690 & 33.62545 & 0.01319 & -0.00539 & 600.570 \\
\hline \multirow[t]{6}{*}{ \# } & 55329.765239 & 33.52162 & 0.02340 & -0.00788 & 600.570 \\
\hline & 55329.774717 & 33.44389 & 0.01553 & -0.07355 & 600.570 \\
\hline & 55390.460520 & 35.06057 & 0.00926 & -0.01522 & 600.570 \\
\hline & 55390.469906 & 34.95219 & 0.00989 & -0.00022 & 600.570 \\
\hline & 55390.479280 & 34.84649 & 0.01008 & -0.03039 & 600.570 \\
\hline & 55390.488654 & 34.78109 & 0.01226 & -0.02240 & 600.570 \\
\hline \# & 55390.498133 & 34.71316 & 0.02654 & -0.04848 & 600.570 \\
\hline \# & 55390.507553 & 34.55764 & 0.05742 & -0.07601 & 600.570 \\
\hline \# & 55390.516939 & 34.51794 & 0.04491 & -0.25450 & 600.571 \\
\hline \# & 55390.526325 & 34.39778 & 0.03611 & -0.05093 & 600.571 \\
\hline \# & 55390.535699 & 34.24055 & 0.02948 & 0.01679 & 600.570 \\
\hline \multirow[t]{27}{*}{ \# } & 55390.545073 & 34.14585 & 0.02327 & -0.00550 & 600.571 \\
\hline & 55390.554725 & 34.04639 & 0.01295 & -0.05073 & 600.570 \\
\hline & 55390.564100 & 33.96213 & 0.01171 & -0.05840 & 600.570 \\
\hline & 55390.573474 & 33.85961 & 0.01183 & -0.03258 & 600.570 \\
\hline & 55390.582952 & 33.78040 & 0.01189 & -0.02163 & 600.570 \\
\hline & 55390.592500 & 33.67088 & 0.01068 & -0.02565 & 600.570 \\
\hline & 55390.601863 & 33.56572 & 0.01378 & -0.06646 & 600.570 \\
\hline & 55390.611249 & 33.48312 & 0.01541 & -0.03775 & 600.570 \\
\hline & 55391.523964 & 25.74355 & 0.01168 & 0.00418 & 600.570 \\
\hline & 55629.824349 & 26.70333 & 0.01020 & -0.00577 & 600.582 \\
\hline & 55635.796335 & 23.62243 & 0.01281 & 0.00327 & 600.580 \\
\hline & 55644.691444 & 38.61303 & 0.00849 & 0.00907 & 600.580 \\
\hline & 55646.783167 & 40.68356 & 0.00941 & -0.02779 & 600.580 \\
\hline & 55666.518971 & 44.39380 & 0.01250 & 0.01109 & 600.661 \\
\hline & 55667.592221 & 35.34860 & 0.00960 & -0.00970 & 600.581 \\
\hline & 55667.601631 & 35.25073 & 0.00937 & -0.04526 & 600.581 \\
\hline & 55667.611017 & 35.16217 & 0.00950 & -0.04016 & 600.581 \\
\hline & 55667.620404 & 35.07056 & 0.00973 & -0.03097 & 600.581 \\
\hline & 55667.629790 & 34.96315 & 0.00926 & -0.01371 & 600.581 \\
\hline & 55667.639292 & 34.86310 & 0.00890 & -0.00189 & 600.601 \\
\hline & 55667.648795 & 34.78823 & 0.00923 & -0.03870 & 600.581 \\
\hline & 55667.658181 & 34.67728 & 0.00918 & -0.01658 & 600.642 \\
\hline & 55667.667626 & 34.58564 & 0.00859 & -0.00233 & 600.581 \\
\hline & 55667.677163 & 34.48485 & 0.00882 & -0.00674 & 600.662 \\
\hline & 55667.686561 & 34.38737 & 0.00882 & -0.01188 & 600.581 \\
\hline & 55667.695936 & 34.25795 & 0.00907 & -0.00941 & 600.581 \\
\hline & 55667.705322 & 34.13713 & 0.00974 & -0.04686 & 600.581 \\
\hline
\end{tabular}

Notes. Points excluded from the analysis are marked by \#. 
A\&A 549, A18 (2013)

Table A.3. continued.

\begin{tabular}{ccccc}
\hline \hline $\begin{array}{c}\text { JDB-2 } 400000 \\
\text { day }\end{array}$ & $\begin{array}{c}\mathrm{RV} \\
\mathrm{m} \mathrm{s}^{-1}\end{array}$ & $\begin{array}{c}1 \sigma \text { error } \\
\mathrm{m} \mathrm{s}^{-1}\end{array}$ & $\begin{array}{c}\text { Bissector span } \\
\mathrm{m} \mathrm{s}^{-1}\end{array}$ & $\begin{array}{c}\text { Exposition time } \\
\mathrm{s}\end{array}$ \\
\hline 55667.714720 & 34.05206 & 0.00972 & -0.02112 & 600.581 \\
55667.724107 & 33.93877 & 0.00968 & -0.00701 & 600.641 \\
55667.733494 & 33.87283 & 0.00979 & -0.03358 & 600.581 \\
55667.742880 & 33.76460 & 0.00968 & -0.01320 & 600.641 \\
55667.752313 & 33.68215 & 0.00910 & 0.01805 & 600.581 \\
55667.761699 & 33.58665 & 0.00940 & 0.01132 & 600.621 \\
55667.773401 & 33.46342 & 0.01030 & -0.02990 & 601.570 \\
55667.782799 & 33.33829 & 0.00983 & -0.05807 & 601.671 \\
55667.794419 & 33.25856 & 0.01239 & -0.00666 & 601.570 \\
55667.804060 & 33.14646 & 0.01293 & -0.02891 & 601.691 \\
55667.813459 & 33.04706 & 0.01229 & -0.00366 & 601.570 \\
55667.824049 & 32.95409 & 0.00980 & -0.01739 & 800.795 \\
55667.835924 & 32.81781 & 0.00934 & -0.01553 & 800.755 \\
55667.847648 & 32.70209 & 0.00989 & -0.03184 & 800.836 \\
55668.611754 & 26.18419 & 0.00668 & -0.00885 & 800.755 \\
\hline
\end{tabular}

